\title{
INJECTIVITY THEOREM FOR PSEUDO-EFFECTIVE LINE BUNDLES AND ITS APPLICATIONS
}

\author{
OSAMU FUJINO AND SHIN-ICHI MATSUMURA
}

Dedicated to Professor Ichiro Enoki on the occasion of his retirement

\begin{abstract}
We formulate and establish a generalization of Kollár's injectivity theorem for adjoint bundles twisted by suitable multiplier ideal sheaves. As applications, we generalize Kollár's torsion-freeness, Kollár's vanishing theorem, and a generic vanishing theorem for pseudo-effective line bundles. Our approach is not Hodge theoretic but analytic, which enables us to treat singular Hermitian metrics with nonalgebraic singularities. For the proof of the main injectivity theorem, we use $L^{2}$-harmonic forms on noncompact Kähler manifolds. For applications, we prove a Bertini-type theorem on the restriction of multiplier ideal sheaves to general members of free linear systems.
\end{abstract}

\section{Contents}

1. Introduction

1.1. Main results

2. Preliminaries

3. Restriction lemma

4. Proof of Proposition 1.9

5. Proof of Theorem $\mathrm{A}$

6. Twists by Nakano semipositive vector bundles

Acknowledgments

References

\section{INTRODUCTION}

The Kodaira vanishing theorem $\overline{K o d}$ is one of the most celebrated results in complex geometry, and it has been generalized to several significant results; for example, the Kawamata-Viehweg vanishing theorem, the Nadel vanishing theorem, Kollár's injectivity theorem (see [F9, Chapter 3]). Kodaira's original proof is based on the theory of harmonic (differential) forms, and has currently been

Received by the editors May 7, 2019, and, in revised form, June 15, 2021, and June 25, 2021. 2020 Mathematics Subject Classification. Primary 32L10; Secondary 32Q15.

Key words and phrases. Injectivity theorems, vanishing theorems, pseudo-effective line bundles, singular Hermitian metrics, multiplier ideal sheaves.

The first author was partially supported by Grant-in-Aid for Young Scientists (A) 24684002 from JSPS and by JSPS KAKENHI Grant Numbers JP16H03925, JP16H06337, JP19H01787, JP20H00111, JP21H00974. The second author was partially supported by Grand-in-Aid for Young Scientists (A) $\sharp 17$ H04821, Grand-in-Aid for Scientific Research (B) $\sharp 21 H 00976$, and Fostering Joint International Research $(\mathrm{A}) \sharp 19 \mathrm{KK} 0342$ from JSPS. 
developed to two approaches from different perspectives: One is the Hodge theoretic approach, which is algebro-geometric theory based on Hodge structures and spectral sequences. The other is the transcendental approach, which is an analytic theory focusing on harmonic forms and $L^{2}$-methods for $\bar{\partial}$-equations. These approaches have been nourishing each other in the last decades.

As is well known, the Kawamata-Viehweg vanishing theorem plays a crucial role in the theory of minimal models for higher-dimensional complex algebraic varieties with only mild singularities. Now some generalizations of Kollár's injectivity theorem allow us to extend the framework of the minimal model program to highly singular varieties (see [A1], A2, [EV], [F1], [F2, [F3, [F6], [F7], [F8, [F9], [F10], [F12, F13, F14]). The reader can find various vanishing theorems and their applications in the minimal model program in [F9, Chapters 3 and 6]. Kollár's original injectivity theorem, which is one of the most important generalizations of the Kodaira vanishing theorem, was first established by using the Hodge theory (see [Kol1]). The following theorem, which is a special case of [F9, Theorem 3.16.2], is obtained from the theory of mixed Hodge structures on cohomology with compact support.

Theorem 1.1 (Injectivity theorem for $\log$ canonical pairs). Let $D$ be a simple normal crossing divisor on a smooth projective variety $X$ and $F$ be a semiample line bundle on $X$. Let $s$ be a nonzero global section of a positive multiple $F^{\otimes m}$ such that the zero locus $s^{-1}(0)$ contains no log canonical centers of the log canonical pair $(X, D)$. Then the map

$$
\times s: H^{i}\left(X, K_{X} \otimes D \otimes F\right) \rightarrow H^{i}\left(X, K_{X} \otimes D \otimes F^{\otimes m+1}\right)
$$

induced by $\otimes s$ is injective for every $i$. Here $K_{X}$ denotes the canonical bundle of $X$.

The Hodge theoretic approach for Theorem 1.1 is algebro-geometric. For the proof, we first take a suitable resolution of singularities, and then take a cyclic cover. After that, we apply the $E_{1}$-degeneration of a Hodge to de Rham type spectral sequence coming from the theory of mixed Hodge structures on cohomology with compact support. In this proof, we do not directly use analytic arguments; on the contrary, we have no analytic proof for Theorem 1.1. This indicates that a precise relation between the Hodge theoretic approach and the transcendental method is not clear yet and is still mysterious. There is room for further research from the analytic viewpoint. In this paper, we pursue the transcendental approach for vanishing theorems instead of the Hodge theoretic approach.

A transcendental approach for Kollár's important work (see Kol1) was first given by Enoki, which improves Kollár's original injectivity theorem to semipositive line bundles on compact Kähler manifolds as an easy application of the theory of harmonic forms. After Enoki's work, several authors obtained some generalizations of Kollár's injectivity theorem from the analytic viewpoint, based on the theory of $L^{2}$-harmonic forms (see, for example, [En], Ta, O3], [F4, [F5], MaS1, MaS2], and MaS4]). Based on the same philosophy, it is natural to expect Theorem 1.1 to hold in the complex analytic setting. However, as we mentioned above, there is no analytic proof for Theorem 1.1. Difficulties lie in that the usual $L^{2}$-method does not work for log canonical singularities, and that no transcendental methods are corresponding to the theory of mixed Hodge structures (see [MaS8, No, LRW] for some approaches). The transcendental method often provides some powerful tools not only in complex geometry but also in algebraic geometry. Therefore it 
is of interest to study various vanishing theorems and related topics by using the transcendental method.

In this paper, by developing the transcendental approach for vanishing theorems, we prove Kollár's injectivity, vanishing, torsion-free theorems, and a generic vanishing theorem for $K_{X} \otimes F \otimes \mathcal{J}(h)$, where $K_{X}$ is the canonical bundle of $X$, $F$ is a pseudo-effective line bundle on $X$, and $\mathcal{J}(h)$ is the multiplier ideal sheaf associated with a singular Hermitian metric $h$. More precisely, this paper contains three main contributions: The first contribution is to prove a generalization of Kollár's injectivity theorem for adjoint bundles twisted by suitable multiplier ideal sheaves (Theorem $\mathrm{A}$ ). The second contribution is to establish a Bertini-type theorem on the restriction of multiplier ideal sheaves (Theorem 1.10). Theorem 1.10 provides a useful tool and enables us to use the inductive argument on dimension. The third contribution is to deduce various results related to vanishing theorems as applications of Theorem 1.10 and Theorem $\mathrm{A}$ (Theorems $\mathrm{B}, \mathrm{C}, \mathrm{D}, \mathrm{E}$, and $\mathrm{F}$ ). Since we adopt the transcendental method, we can formulate all the results for singular Hermitian metrics and (quasi-)plurisubharmonic functions with arbitrary singularities. This is one of the main advantages of our approach in this paper. The Hodge theoretic approach explained before does not work for singular Hermitian metrics with nonalgebraic singularities. Furthermore, we sometimes have to deal with singular Hermitian metrics with nonalgebraic singularities for several important applications in birational geometry even when we consider problems in algebraic geometry (see, for example, $[\mathrm{Si}], \mathrm{Pa}], \mathrm{DHP}, \mathrm{GM}]$, and [LP]). Therefore, it is worth formulating and proving various results for singular Hermitian metrics with arbitrary singularities although they are much more complicated than singular Hermitian metrics with only algebraic singularities.

1.1. Main results. Here, we explain the main results of this paper (Theorems A, B, C, D, E, E, and Theorem 1.10). Theorem A and Theorem 1.10 play important roles in this paper, and other results follow from Theorem $\mathrm{A}$ and Theorem 1.10 (see Proposition [1.9). We first recall the definition of pseudo-effective line bundles on compact complex manifolds.

Definition 1.2 (Pseudo-effective line bundles). Let $F$ be a holomorphic line bundle on a compact complex manifold $X$. We say that $F$ is pseudo-effective if there exists a singular Hermitian metric $h$ on $F$ with $\sqrt{-1} \Theta_{h}(F) \geq 0$. When $X$ is projective, it is well known that $F$ is pseudo-effective if and only if $F$ is pseudo-effective in the usual sense, that is, $F^{\otimes m} \otimes H$ is big for any ample line bundle $H$ on $X$ and any positive integer $m$.

The first result is an Enoki-type injectivity theorem.

Theorem A (Enoki-type injectivity). Let $F$ be a holomorphic line bundle on a compact Kähler manifold $X$ and let $h$ be a singular Hermitian metric on $F$. Let $M$ be a holomorphic line bundle on $X$ and let $h_{M}$ be a smooth Hermitian metric on M. Assume that

$$
\sqrt{-1} \Theta_{h_{M}}(M) \geq 0 \quad \text { and } \quad \sqrt{-1}\left(\Theta_{h}(F)-t \Theta_{h_{M}}(M)\right) \geq 0
$$

for some $t>0$. Let $s$ be a nonzero global section of $M$. Then the map

$$
\times s: H^{i}\left(X, K_{X} \otimes F \otimes \mathcal{J}(h)\right) \rightarrow H^{i}\left(X, K_{X} \otimes F \otimes \mathcal{J}(h) \otimes M\right)
$$

induced by $\otimes s$ is injective for every $i$, where $K_{X}$ is the canonical bundle of $X$ and $\mathcal{J}(h)$ is the multiplier ideal sheaf of $h$. 
Remark 1.3. Let $L$ be a semipositive line bundle on $X$, that is, it admits a smooth Hermitian metric with semipositive curvature. Let $F=L^{\otimes m}$ and $M=L^{\otimes k}$ for positive integers $m$ and $k$. Then we obtain Enoki's original injectivity theorem (see [En, Theorem 0.2]) from Theorem $\mathrm{A}$

In the case of $M=F$, Theorem $\mathrm{A}$ has been proved in MaS4 under the assumption $\sup _{X}|s|_{h}<\infty$. This assumption is a natural condition to guarantee that the multiplication map $\times s$ is well-defined. However, for our applications in this paper, we need to formulate Theorem $\mathrm{A}$ for a different $\left(M, h_{M}\right)$ from $(F, h)$. This formulation, which may look slightly artificial, is quite powerful and can produce applications, but raises a new difficulty in the proof: the set of points $x \in X$ with $\nu(h, x)>0$ is not necessarily contained in a proper Zariski closed set, although such a situation was excluded in [MaS4] thanks to the assumption $\sup _{X}|s|_{h}<\infty$, where $\nu(h, x)$ denotes the Lelong number of the local weight of $h$ at $x$. Compared to MaS4, Theorem $\mathrm{A}$ is novel in the technique to overcome this difficulty (see Section [5] for the technical details), and further, it will be generalized to certain noncompact manifolds along with other techniques (see [MaS5]). Note that Theorem $\mathrm{A}$ can be seen as a generalization not only of Enoki's injectivity theorem but also of the Nadel vanishing theorem. In Section 4 we will explain how to reduce Demailly's original formulation of the Nadel vanishing theorem (see Theorem 1.4 below) to Theorem $\mathrm{A}$ for the reader's convenience.

Theorem 1.4 (Nadel vanishing theorem due to Demailly: D2, Theorem 4.5]). Let $V$ be a smooth projective variety equipped with a Kähler form $\omega$. Let $L$ be a holomorphic line bundle on $V$ and let $h_{L}$ be a singular Hermitian metric on $L$ such that $\sqrt{-1} \Theta_{h_{L}}(L) \geq \varepsilon \omega$ for some $\varepsilon>0$. Then

$$
H^{i}\left(V, K_{V} \otimes L \otimes \mathcal{J}\left(h_{L}\right)\right)=0
$$

for every $i>0$, where $K_{V}$ is the canonical bundle of $V$ and $\mathcal{J}\left(h_{L}\right)$ is the multiplier ideal sheaf of $h_{L}$.

A semiample line bundle is always semipositive. Thus, as a direct consequence of Theorem A, we obtain Theorem B which is a generalization of Kollár's original injectivity theorem (see [Kol1]).

Theorem B (Kollár-type injectivity). Let $F$ be a holomorphic line bundle on a compact Kähler manifold $X$ and let $h$ be a singular Hermitian metric on $F$ such that $\sqrt{-1} \Theta_{h}(F) \geq 0$. Let $N_{1}$ and $N_{2}$ be semiample line bundles on $X$ and let $s$ be a nonzero global section of $N_{2}$. Assume that $N_{1}^{\otimes a} \simeq N_{2}^{\otimes b}$ for some positive integers $a$ and $b$. Then the map

$$
\times s: H^{i}\left(X, K_{X} \otimes F \otimes \mathcal{J}(h) \otimes N_{1}\right) \rightarrow H^{i}\left(X, K_{X} \otimes F \otimes \mathcal{J}(h) \otimes N_{1} \otimes N_{2}\right)
$$

induced by $\otimes s$ is injective for every $i$, where $K_{X}$ is the canonical bundle of $X$ and $\mathcal{J}(h)$ is the multiplier ideal sheaf of $h$.

Remark 1.5 .

(1) Let $X$ be a smooth projective variety and $(F, h)$ be a trivial Hermitian line bundle. Then we obtain Kollár's original injectivity theorem (see Kol1, Theorem 2.2]) from Theorem $B$. 
(2) For the proof of Theorem [B, we may assume that $b=1$, that is, $N_{2} \simeq N_{1}^{\otimes a}$ by replacing $s$ with $s^{b}$. We note that the composition

$$
\begin{aligned}
H^{i}\left(X, K_{X} \otimes F \otimes \mathcal{J}(h) \otimes N_{1}\right) & \stackrel{\times s}{\longrightarrow} H^{i}\left(X, K_{X} \otimes F \otimes \mathcal{J}(h) \otimes N_{1} \otimes N_{2}\right) \\
& \stackrel{\times s^{b-1}}{\longrightarrow} H^{i}\left(X, K_{X} \otimes F \otimes \mathcal{J}(h) \otimes N_{1} \otimes N_{2}^{\otimes b}\right)
\end{aligned}
$$

is the map $\times s^{b}$ induced by $\otimes s^{b}$.

Theorem C is a generalization of Kollár's torsion-free theorem and Theorem D is a generalization of Kollár's vanishing theorem (see [Kol1, Theorem 2.1]).

Theorem C (Kollár-type torsion-freeness). Let $f: X \rightarrow Y$ be a surjective morphism from a compact Kähler manifold $X$ onto a projective variety $Y$. Let $F$ be a holomorphic line bundle on $X$ and let $h$ be a singular Hermitian metric on $F$ such that $\sqrt{-1} \Theta_{h}(F) \geq 0$. Then

$$
R^{i} f_{*}\left(K_{X} \otimes F \otimes \mathcal{J}(h)\right)
$$

is torsion-free for every $i$, where $K_{X}$ is the canonical bundle of $X$ and $\mathcal{J}(h)$ is the multiplier ideal sheaf of $h$.

Theorem D (Kollár-type vanishing theorem). Let $f: X \rightarrow Y$ be a surjective morphism from a compact Kähler manifold $X$ onto a projective variety $Y$. Let $F$ be a holomorphic line bundle on $X$ and let $h$ be a singular Hermitian metric on $F$ such that $\sqrt{-1} \Theta_{h}(F) \geq 0$. Let $N$ be a holomorphic line bundle on $X$. We assume that there exist positive integers $a$ and $b$ and an ample line bundle $H$ on $Y$ such that $N^{\otimes a} \simeq f^{*} H^{\otimes b}$. Then we obtain that

$$
H^{i}\left(Y, R^{j} f_{*}\left(K_{X} \otimes F \otimes \mathcal{J}(h) \otimes N\right)\right)=0
$$

for every $i>0$ and $j$, where $K_{X}$ is the canonical bundle of $X$ and $\mathcal{J}(h)$ is the multiplier ideal sheaf of $h$.

Remark 1.6.

(1) If $X$ is a smooth projective variety and $(F, h)$ is trivial, then Theorem $\mathrm{C}$ is nothing but Kollár's torsion-free theorem. Furthermore, if $N \simeq f^{*} H$, that is, $a=b=1$, then Theorem $\mathrm{D}$ is the Kollár vanishing theorem. For the details, see Kol1, Theorem 2.1].

(2) There exists a clever proof of Kollár's torsion-freeness by the theory of variations of Hodge structure (see $\mathrm{Ar}$ ).

(3) In MaS6], the second author obtained a natural analytic generalization of Kollár's vanishing theorem, which corresponds to the case where $h$ is a smooth Hermitian metric and contains Ohsawa's vanishing theorem (see [O2]) as a special case.

(4) In F15, the first author proved a vanishing theorem containing both Theorem 1.4 and Theorem $\mathrm{D}$ as special cases, which is called the vanishing theorem of Kollár-Nadel type.

By combining Theorem D with the Castelnuovo-Mumford regularity, we can easily obtain Corollary 1.7, which is a complete generalization of [Hö, Lemma 3.35 and Remark 3.36]. The proof of [Hö, Lemma 3.35] depends on a generalization of the Ohsawa-Takegoshi $L^{2}$ extension theorem. We note that Höring claims the weak positivity of $f_{*}\left(K_{X / Y} \otimes F\right)$ under some extra assumptions by using [Hö, Lemma 3.35]. For the details, see [Hö, 3.H Multiplier ideals]. 
Corollary 1.7. Let $f: X \rightarrow Y$ be a surjective morphism from a compact Kähler manifold $X$ onto a projective variety $Y$. Let $F$ be a holomorphic line bundle on $X$ and let $h$ be a singular Hermitian metric on $F$ such that $\sqrt{-1} \Theta_{h}(F) \geq 0$. Let $H$ be an ample and globally generated line bundle on $Y$. Then

$$
R^{i} f_{*}\left(K_{X} \otimes F \otimes \mathcal{J}(h)\right) \otimes H^{\otimes m}
$$

is globally generated for every $i \geq 0$ and $m \geq \operatorname{dim} Y+1$, where $K_{X}$ is the canonical bundle of $X$ and $\mathcal{J}(h)$ is the multiplier ideal sheaf of $h$.

As a direct consequence of Theorem D, we obtain Theorem E, See Definition 1.8 for the definition of GV-sheaves in the sense of Pareschi and Popa and see [Sc, Theorem 25.5 and Definition 26.3] for the details of GV-sheaves.

Theorem E (GV-sheaves). Let $f: X \rightarrow A$ be a morphism from a compact Kähler manifold $X$ to an Abelian variety $A$. Let $F$ be a holomorphic line bundle on $X$ and let $h$ be a singular Hermitian metric on $F$ such that $\sqrt{-1} \Theta_{h}(F) \geq 0$. Then

$$
R^{i} f_{*}\left(K_{X} \otimes F \otimes \mathcal{J}(h)\right)
$$

is a $G V$-sheaf for every $i$, where $K_{X}$ is the canonical bundle of $X$ and $\mathcal{J}(h)$ is the multiplier ideal sheaf of $h$.

Definition 1.8 (GV-sheaves in the sense of Pareschi and Popa: $\mathrm{PP}$ ). Let $A$ be an Abelian variety. A coherent sheaf $\mathcal{F}$ on $A$ is said to be a GV-sheaf if

$$
\operatorname{codim}_{\mathrm{Pic}^{0}(A)}\left\{L \in \operatorname{Pic}^{0}(A) \mid H^{i}(A, \mathcal{F} \otimes L) \neq 0\right\} \geq i
$$

for every $i$.

The final one is a generalization of the generic vanishing theorem (see GL, $\mathrm{Ha}$, $[\mathrm{PP}]$ ). The formulation of Theorem $\mathrm{F}$ is closer to $[\mathrm{Ha}$ and $[\mathrm{PP}$ than to the original generic vanishing theorem by Green and Lazarsfeld in GL].

Theorem $\mathbf{F}$ (Generic vanishing theorem). Let $f: X \rightarrow A$ be a morphism from a compact Kähler manifold $X$ to an Abelian variety $A$. Let $F$ be a holomorphic line bundle on $X$ and let $h$ be a singular Hermitian metric on $F$ such that $\sqrt{-1} \Theta_{h}(F) \geq$ 0. Then

$$
\begin{aligned}
\operatorname{codim}_{\mathrm{Pic}^{0}(A)}\left\{L \in \operatorname{Pic}^{0}(A) \mid H^{i}\left(X, K_{X} \otimes F \otimes \mathcal{J}(h) \otimes f^{*} L\right)\right. & \neq 0\} \\
& \geq i-(\operatorname{dim} X-\operatorname{dim} f(X))
\end{aligned}
$$

for every $i \geq 0$, where $K_{X}$ is the canonical bundle of $X$ and $\mathcal{J}(h)$ is the multiplier ideal sheaf of $h$.

The main results explained above are closely related to each other. The following proposition, which is also one of the main contributions in this paper, shows several relations among them. From Proposition 1.9, we see that it is sufficient to prove Theorem A. The proof of Proposition 1.9 will be given in Section 4

Proposition 1.9. We have the following relations among the above theorems.

(i) Theorem $\mathrm{A}$ implies Theorem $\mathrm{B}$.

(ii) Theorem $\mathrm{B}$ is equivalent to Theorem $\mathrm{C}$ and Theorem $\mathrm{D}$

(iii) Theorem $\mathrm{D}$ implies Theorem $\mathrm{E}$,

(iv) Theorem $\mathrm{C}$ and Theorem $\mathrm{E}$ imply Theorem $\mathrm{E}$. 
A key ingredient of Proposition 1.9 is the following theorem, which can be seen as a Bertini-type theorem on the restriction of multiplier ideal sheaves to general members of free linear systems. Theorem 1.10 enables us to use the inductive argument on dimension. We remark that $\mathcal{G}$ in Theorem 1.10 is not always an intersection of countably many Zariski open sets (see Example 3.10). The proof of Theorem 1.10, which is quite technical, will be given in Section 3

Theorem 1.10 (Density of good divisors: Theorem [3.6). Let $X$ be a compact complex manifold, let $\Lambda$ be a free linear system on $X$ with $\operatorname{dim} \Lambda \geq 1$, and let $\varphi$ be a quasi-plurisubharmonic function on $X$. We put

$$
\mathcal{G}:=\left\{H \in \Lambda \mid H \text { is smooth and } \mathcal{J}\left(\left.\varphi\right|_{H}\right)=\left.\mathcal{J}(\varphi)\right|_{H}\right\} .
$$

Then $\mathcal{G}$ is dense in $\Lambda$ in the classical topology, that is, the Euclidean topology.

Although the above formulation is sufficient for our applications, it is of independent interest to find a more precise formulation. The following problem, posed by Sébastien Boucksom, is reasonable from the viewpoint of Berndtsson's complex Prekopa theorem (see $[\mathrm{Be}]$ ).

Problem 1.11. In Theorem 1.10 is the complement $\Lambda \backslash \mathcal{G}$ a pluripolar subset of $\Lambda$ ?

All the results explained above hold even if we replace $K_{X}$ with $K_{X} \otimes E$, where $E$ is any Nakano semipositive vector bundle on $X$. We will explain Theorem 1.12 in Section 6 .

Theorem 1.12 (Twists by Nakano semipositive vector bundles). Let E be a Nakano semipositive vector bundle on a compact Kähler manifold $X$. Then Theorems $\mathrm{A}$, B, L, D, E, E, Theorem 1.4, Corollary 1.7, and Proposition 1.9 hold even when $K_{X}$ is replaced with $K_{X} \otimes E$.

In this paper, we assume that all the varieties and manifolds are compact and connected for simplicity. We summarize the contents of this paper. In Section 2, we recall some basic definitions and collect several preliminary lemmas. Section 3 is devoted to the proof of Theorem 1.10. Theorem 1.10 plays a crucial role in the proof of Proposition 1.9. In Section 4 we prove Proposition 1.9 and Corollary 1.7. and explain how to reduce Theorem 1.4 to Theorem $\mathrm{A}$. By these results, we see that all we have to do is to establish Theorem A. In Section 5, we give a detailed proof of Theorem A. In the final section: Section 6. we explain how to modify the arguments used before for the proof of Theorem 1.12

After the authors put a preprint version of this paper on arXiv, some further generalizations of Theorem $\mathrm{A}$ have been studied in [MaS5], CDM], [ZZ], and a relative version of Theorem [1.10 has been established in [F16]. See [Ta], [F5], MaS5, CDM], F16 for some injectivity, torsion-free, and vanishing theorems for noncompact manifolds.

\section{Preliminaries}

We briefly review the definition of singular Hermitian metrics, (quasi-)plurisubharmonic functions, and Nadel's multiplier ideal sheaves. See [D3] for the details. 
Definition 2.1 (Singular Hermitian metrics and curvatures). Let $F$ be a holomorphic line bundle on a complex manifold $X$. A singular Hermitian metric on $F$ is a metric $h$ which is given in every trivialization $\theta:\left.F\right|_{\Omega} \simeq \Omega \times \mathbb{C}$ by

$$
|\xi|_{h}=|\theta(\xi)| e^{-\varphi} \text { on } \Omega,
$$

where $\xi$ is a section of $F$ on $\Omega$ and $\varphi \in L_{\text {loc }}^{1}(\Omega)$ is an arbitrary function. Here $L_{\text {loc }}^{1}(\Omega)$ is the space of locally integrable functions on $\Omega$. We usually call $\varphi$ the weight function of the metric with respect to the trivialization $\theta$. The curvature of a singular Hermitian metric $h$ is defined by

$$
\sqrt{-1} \Theta_{h}(F):=2 \sqrt{-1} \partial \bar{\partial} \varphi
$$

where $\varphi$ is a weight function and $\sqrt{-1} \partial \bar{\partial} \varphi$ is taken in the sense of currents. It is easy to see that the right-hand side does not depend on the choice of trivializations.

The notion of multiplier ideal sheaves introduced by Nadel plays an important role in the recent developments of complex geometry and algebraic geometry.

Definition 2.2 ((Quasi-)plurisubharmonic functions and multiplier ideal sheaves). A function $u: \Omega \rightarrow[-\infty, \infty)$ defined on an open set $\Omega \subset \mathbb{C}^{n}$ is said to be plurisubharmonic if

- $u$ is upper semicontinuous, and

- for every complex line $L \subset \mathbb{C}^{n}$, the restriction $\left.u\right|_{\Omega \cap L}$ to $L$ is subharmonic on $\Omega \cap L$, that is, for every $a \in \Omega$ and $\xi \in \mathbb{C}^{n}$ satisfying $|\xi|<d\left(a, \Omega^{c}\right)$, the function $u$ satisfies the mean inequality

$$
u(a) \leq \frac{1}{2 \pi} \int_{0}^{2 \pi} u\left(a+e^{i \theta} \xi\right) d \theta
$$

Let $X$ be a complex manifold. A function $\varphi: X \rightarrow[-\infty, \infty)$ is said to be plurisubharmonic on $X$ if there exists an open cover $\left\{U_{i}\right\}_{i \in I}$ of $X$ such that $\varphi_{U_{i}}$ is plurisubharmonic on $U_{i}\left(\subset \mathbb{C}^{n}\right)$ for every $i$. We can easily see that this definition is independent of the choice of open covers. A quasi-plurisubharmonic function is a function $\varphi$ which is locally equal to the sum of a plurisubharmonic function and of a smooth function. If $\varphi$ is a quasi-plurisubharmonic function on a complex manifold $X$, then the multiplier ideal sheaf $\mathcal{J}(\varphi) \subset \mathcal{O}_{X}$ is defined by

$$
\Gamma(U, \mathcal{J}(\varphi)):=\left\{\left.f \in \mathcal{O}_{X}(U)|| f\right|^{2} e^{-2 \varphi} \in L_{\text {loc }}^{1}(U)\right\}
$$

for every open set $U \subset X$. Then it is known that $\mathcal{J}(\varphi)$ is a coherent ideal sheaf (see, for example, D3, (5.7) Lemma]). Let $S$ be a complex submanifold of $X$. Then the restriction $\left.\mathcal{J}(\varphi)\right|_{S}$ of the multiplier ideal sheaf $\mathcal{J}(\varphi)$ to $S$ is defined by the image of $\mathcal{J}(\varphi)$ under the natural surjective morphism $\mathcal{O}_{X} \rightarrow \mathcal{O}_{S}$, that is,

$$
\left.\mathcal{J}(\varphi)\right|_{S}=\mathcal{J}(\varphi) / \mathcal{J}(\varphi) \cap \mathcal{I}_{S},
$$

where $\mathcal{I}_{S}$ is the defining ideal sheaf of $S$ on $X$. We note that the restriction $\left.\mathcal{J}(\varphi)\right|_{S}$ does not always coincide with $\mathcal{J}(\varphi) \otimes \mathcal{O}_{S}=\mathcal{J}(\varphi) / \mathcal{J}(\varphi) \cdot \mathcal{I}_{S}$.

We have already used $\mathcal{J}(h)$ in theorems in Section 1

Definition 2.3. Let $F$ be a holomorphic line bundle on a complex manifold $X$ and let $h$ be a singular Hermitian metric on $F$. We assume $\sqrt{-1} \Theta_{h}(F) \geq \gamma$ for some smooth $(1,1)$-form $\gamma$ on $X$. We fix a smooth Hermitian metric $h_{\infty}$ on $F$. Then 
we can write $h=h_{\infty} e^{-2 \psi}$ for some $\psi \in L_{\text {loc }}^{1}(X)$. Then $\psi$ coincides with a quasiplurisubharmonic function $\varphi$ on $X$ almost everywhere. We define the multiplier ideal sheaf $\mathcal{J}(h)$ of $h$ by $\mathcal{J}(h):=\mathcal{J}(\varphi)$.

We close this section with the following lemmas, which will be used in the proof of Theorem $\mathrm{A}$ in Section 5 .

Lemma 2.4 ([01, Proposition 1.1]). Let $\omega$ and $\widetilde{\omega}$ be positive $(1,1)$-forms on an $n$ dimensional complex manifold with $\widetilde{\omega} \geq \omega$. If $u$ is an $(n, q)$-form, then $|u|_{\widetilde{\omega}}^{2} d V_{\widetilde{\omega}} \leq$ $|u|_{\omega}^{2} d V_{\omega}$. Furthermore, if $u$ is an $(n, 0)$-form, then $|u|_{\widetilde{\omega}}^{2} d V_{\widetilde{\omega}}=|u|_{\omega}^{2} d V_{\omega}$. Here $|u|_{\omega}$ (resp. $\left.|u|_{\widetilde{\omega}}\right)$ is the pointwise norm of $u$ with respect to $\omega$ (resp. $\widetilde{\omega}$ ) and $d V_{\omega}$ (resp. $\left.d V_{\widetilde{\omega}}\right)$ is the volume form defined by $d V_{\omega}:=\omega^{n} / n !\left(\right.$ resp. $\left.d V_{\widetilde{\omega}}:=\widetilde{\omega}^{n} / n !\right)$.

Proof. This lemma follows from simple computations. Thus, we omit the proof.

Lemma 2.5. Let $\varphi: \mathcal{H}_{1} \rightarrow \mathcal{H}_{2}$ be a bounded operator (continuous linear map) between Hilbert spaces $\mathcal{H}_{1}, \mathcal{H}_{2}$. If $\left\{w_{k}\right\}_{k=1}^{\infty}$ weakly converges to $w$ in $\mathcal{H}_{1}$, then $\left\{\varphi\left(w_{k}\right)\right\}_{k=1}^{\infty}$ weakly converges to $\varphi(w)$.

Proof. By taking the adjoint operator $\varphi^{*}$, for every $v \in \mathcal{H}_{2}$, we have

$$
\left\langle\varphi\left(w_{k}\right), v\right\rangle_{\mathcal{H}_{2}}=\left\langle\langle w _ { k } , \varphi ^ { * } ( v ) \rangle _ { \mathcal { H } _ { 1 } } \rightarrow \left\langle\left\langle w, \varphi^{*}(v)\right\rangle_{\mathcal{H}_{1}}=\left\langle\langle\varphi(w), v\rangle_{\mathcal{H}_{2}} .\right.\right.\right.
$$

This completes the proof.

Lemma 2.6. Let $L$ be a closed subspace in a Hilbert space $\mathcal{H}$. Then $L$ is closed with respect to the weak topology of $\mathcal{H}$, that is, if a sequence $\left\{w_{k}\right\}_{k=1}^{\infty}$ in $L$ weakly converges to $w$, then the weak limit $w$ belongs to $L$.

Proof. By the orthogonal decomposition, there exists a closed subspace $M$ such that $L=M^{\perp}$. Then it follows that $w \in M^{\perp}=L$ since $0=\left\langle\left\langle w_{k}, v\right\rangle_{\mathcal{H}} \rightarrow\langle w, v\rangle_{\mathcal{H}}\right.$ for any $v \in M$.

\section{RESTRICTION LEMMA}

This section is devoted to the proof of Theorem 1.10 (see Theorem 3.6), which will play a crucial role in the proof of Proposition 1.9. The following lemma is a direct consequence of the Ohsawa-Takegoshi $L^{2}$ extension theorem (see [OT, Theorem]).

Lemma 3.1. Let $X$ be a complex manifold and let $\varphi$ be a quasi-plurisubharmonic function on $X$. We consider a filtration

$$
F_{k} \subset F_{k-1} \subset \cdots \subset F_{1} \subset F_{0}:=X,
$$

where $F_{i}$ is a smooth hypersurface of $F_{i-1}$ for every $i$. Then we obtain that

$$
\left.\left.\left.\mathcal{J}\left(\left.\varphi\right|_{F_{k}}\right) \subset \mathcal{J}\left(\left.\varphi\right|_{F_{k-1}}\right)\right|_{F_{k}} \subset \cdots \subset \mathcal{J}\left(\left.\varphi\right|_{F_{1}}\right)\right|_{F_{k}} \subset \mathcal{J}(\varphi)\right|_{F_{k}} .
$$

Proof. This immediately follows from the Ohsawa-Takegoshi $L^{2}$ extension theorem.

The following lemma is a key ingredient of the proof of Theorem 1.10 (see Theorem 3.6). 
Lemma 3.2. Let $X$ and $\varphi$ be as in Theorem 1.10, Let $H_{i}$ be a Cartier divisor on $X$ for $1 \leq i \leq k$. We assume the following condition:

\$ The divisor $\sum_{i=1}^{k} H_{i}$ is a simple normal crossing divisor on $X$. Moreover, for every $1 \leq i_{1}<i_{2}<\cdots<i_{l} \leq k$ and any $P \in H_{i_{1}} \cap H_{i_{2}} \cap \cdots \cap H_{i_{l}}$, the set $\left\{f_{i_{1}}, f_{i_{2}}, \cdots, f_{i_{l}}\right\}$ is a regular sequence for $\mathcal{O}_{X, P} / \mathcal{J}(\varphi)_{P}$, where $f_{i}$ is a (local) defining equation of $H_{i}$ for every $i$.

Furthermore, we assume that $\mathcal{J}\left(\left.\varphi\right|_{F_{k}}\right)=\left.\mathcal{J}(\varphi)\right|_{F_{k}}$ holds, where $F_{i}:=H_{1} \cap H_{2} \cap$ $\cdots \cap H_{i}$ for $1 \leq i \leq k$. Then for every $j$, the equality $\mathcal{J}\left(\left.\varphi\right|_{F_{j}}\right)=\left.\mathcal{J}(\varphi)\right|_{F_{j}}$ holds on a neighborhood of $F_{k}$ in $F_{j}$.

Before we prove Lemma 3.2, we make some remarks to help the reader understand condition

Remark 3.3.

(1) Let $(A, \mathfrak{m})$ be a local ring and let $M$ be a finitely generated (nonzero) $A$ module. Let $\left\{x_{1}, \ldots, x_{r}\right\}$ be a sequence of elements of $\mathfrak{m}$. We put $M_{0}=M$ and $M_{i}=M / x_{1} M+\cdots+x_{i} M$. Then $\left\{x_{1}, \ldots, x_{r}\right\}$ is said to be a regular sequence for $M$ if $\times x_{i+1}: M_{i} \rightarrow M_{i}$ is injective for every $0 \leq i \leq r-1$.

(2) Condition in Lemma 3.2 does not depend on the order of $\left\{H_{1}, H_{2}, \cdots, H_{k}\right\}$ (see, for example, $\mathrm{MaH}$, Theorem 16.3] and [AK, Chapter III, Corollary (3.5)]).

(3) Let $\mathcal{F}$ be a coherent analytic sheaf on a compact complex manifold $X$. Then there exists a finite family $\left\{Y_{i}\right\}_{i \in I}$ of irreducible analytic subsets of $X$ such that

$$
\operatorname{Ass}_{\mathcal{O}_{X, x}}\left(\mathcal{F}_{x}\right)=\left\{\mathfrak{p}_{x, 1}, \ldots, \mathfrak{p}_{x, r(x)}\right\}
$$

where $\mathfrak{p}_{x, 1}, \ldots, \mathfrak{p}_{x, r(x)}$ are prime ideals of $\mathcal{O}_{X, x}$ associated to the irreducible components of the germs $x \in Y_{i}$ (see, for example [Man, (I.6) Lemma]). Note that $Y_{i}$ is called an analytic subset associated with $\mathcal{F}$. In this paper, we simply say that $Y_{i}$ is an associated prime of $\mathcal{F}$ if there is no risk of confusion. Then condition $\boldsymbol{\phi}$ is equivalent to the following condition:

- The divisor $\sum_{i=1}^{k} H_{i}$ is a simple normal crossing divisor on $X$. Moreover, for every $1 \leq i_{1}<i_{2}<\cdots<i_{l-1}<i_{l} \leq k$, the divisor $H_{i_{l}}$ contains no associated primes of $\mathcal{O}_{X} / \mathcal{J}(\varphi)$ and $\mathcal{O}_{H_{i_{1}} \cap \cdots \cap H_{i_{l-1}}} /\left.\mathcal{J}(\varphi)\right|_{H_{i_{1}} \cap \cdots \cap H_{i_{l-1}}}$.

(4) (3.1) below may be helpful to understand condition $\boldsymbol{\$}$. We put $H_{i_{1} \cdots i_{m}}:=$ $H_{i_{1}} \cap \cdots \cap H_{i_{m}}$ for every $1 \leq i_{1}<\cdots<i_{m} \leq k$. Then we can inductively check that

$$
\left.\left.\left.0 \rightarrow \mathcal{J}(\varphi)\right|_{H_{i_{1} \cdots i_{l-1}}} \otimes \mathcal{O}_{H_{i_{1} \cdots i_{l-1}}}\left(-H_{i_{l}}\right) \rightarrow \mathcal{J}(\varphi)\right|_{H_{i_{1} \cdots i_{l-1}}} \rightarrow \mathcal{J}(\varphi)\right|_{H_{i_{1} \cdots i_{l}}} \rightarrow 0
$$

is exact and that

$$
\begin{aligned}
0 & \rightarrow\left(\mathcal{O}_{H_{i_{1} \cdots i_{l-1}}} /\left.\mathcal{J}(\varphi)\right|_{H_{i_{1} \cdots i_{l}-1}}\right) \otimes \mathcal{O}_{H_{i_{1} \cdots i_{l}-1}}\left(-H_{i_{l}}\right) \\
& \rightarrow \mathcal{O}_{H_{i_{1} \cdots i_{l-1}}} /\left.\mathcal{J}(\varphi)\right|_{H_{i_{1} \cdots i_{l-1}}} \rightarrow \mathcal{O}_{H_{i_{1} \cdots i_{l}}} /\left.\mathcal{J}(\varphi)\right|_{H_{i_{1} \cdots i_{l}}} \rightarrow 0
\end{aligned}
$$

is also exact (see (3.3) and (3.4) in the proof of Lemma 3.2). 
Proof of Lemma 3.2. By condition $\boldsymbol{\$}$, the morphism $\gamma$ in the following commutative diagram is injective.

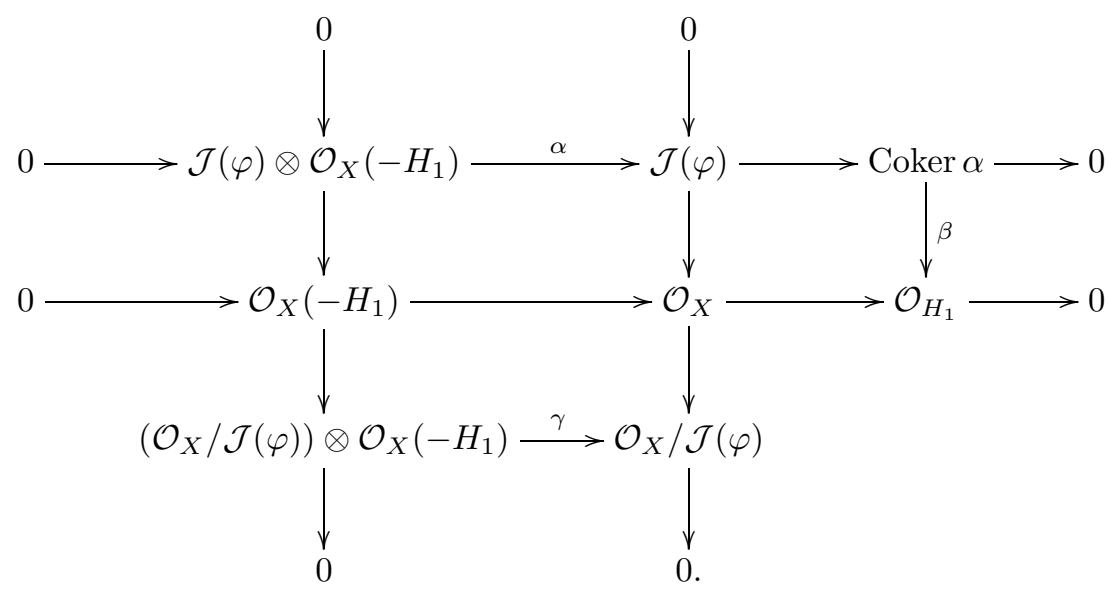

Therefore $\beta$ is also injective. This implies that Coker $\alpha=\left.\mathcal{J}(\varphi)\right|_{H_{1}}$ by definition. Thus, we obtain the following short exact sequence:

$$
\left.0 \rightarrow \mathcal{J}(\varphi) \otimes \mathcal{O}_{X}\left(-H_{1}\right) \rightarrow \mathcal{J}(\varphi) \rightarrow \mathcal{J}(\varphi)\right|_{H_{1}} \rightarrow 0 .
$$

We also obtain the following short exact sequence:

$$
0 \rightarrow\left(\mathcal{O}_{X} / \mathcal{J}(\varphi)\right) \otimes \mathcal{O}_{X}\left(-H_{1}\right) \stackrel{\gamma}{\rightarrow} \mathcal{O}_{X} / \mathcal{J}(\varphi) \rightarrow \mathcal{O}_{H_{1}} /\left.\mathcal{J}(\varphi)\right|_{H_{1}} \rightarrow 0
$$

by the above big commutative diagram. Similarly, by condition $\mathbf{\uparrow}$, we can inductively check that

$$
\left.\left.\left.0 \rightarrow \mathcal{J}(\varphi)\right|_{F_{i}} \otimes \mathcal{O}_{F_{i}}\left(-H_{i+1}\right) \rightarrow \mathcal{J}(\varphi)\right|_{F_{i}} \rightarrow \mathcal{J}(\varphi)\right|_{F_{i+1}} \rightarrow 0
$$

and

$(3.4) 0 \rightarrow\left(\mathcal{O}_{F_{i}} /\left.\mathcal{J}(\varphi)\right|_{F_{i}}\right) \otimes \mathcal{O}_{F_{i}}\left(-H_{i+1}\right) \rightarrow \mathcal{O}_{F_{i}} /\left.\mathcal{J}(\varphi)\right|_{F_{i}} \rightarrow \mathcal{O}_{F_{i+1}} /\left.\mathcal{J}(\varphi)\right|_{F_{i+1}} \rightarrow 0$ are exact for every $1 \leq i \leq k-1$. For $0 \leq i \leq k-1$, we consider the following commutative diagram:

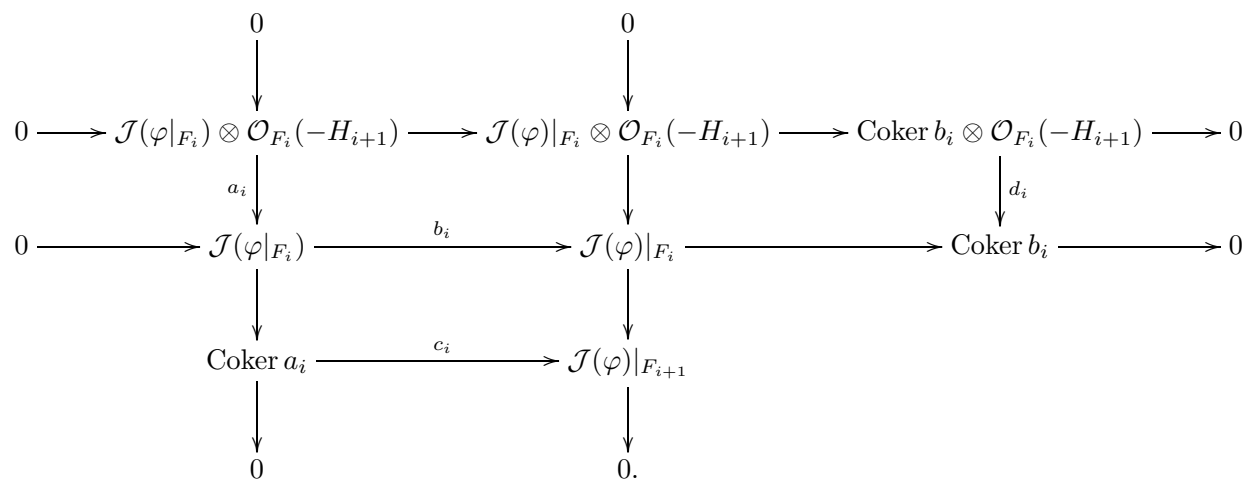

The assumption $\mathcal{J}\left(\left.\varphi\right|_{F_{k}}\right)=\left.\mathcal{J}(\varphi)\right|_{F_{k}}$ implies that $\left.\mathcal{J}\left(\left.\varphi\right|_{F_{k-1}}\right)\right|_{F_{k}}=\left.\mathcal{J}(\varphi)\right|_{F_{k}}$ holds by $\left.\left.\mathcal{J}\left(\left.\varphi\right|_{F_{k}}\right) \subset \mathcal{J}\left(\left.\varphi\right|_{F_{k-1}}\right)\right|_{F_{k}} \subset \cdots \subset \mathcal{J}(\varphi)\right|_{F_{k}}$ in Lemma 3.1. If $\left.\mathcal{J}\left(\left.\varphi\right|_{F_{i}}\right)\right|_{F_{i+1}}=$ $\left.\mathcal{J}(\varphi)\right|_{F_{i+1}}$ on a neighborhood of $F_{k}$ in $F_{i+1}$, then $c_{i}$ is surjective on a neighborhood of $F_{k}$ in $F_{i+1}$ by the definition of $\left.\mathcal{J}\left(\left.\varphi\right|_{F_{i}}\right)\right|_{F_{i+1}}$. Then $d_{i}$ is also surjective on a 
neighborhood of $F_{k}$ in $F_{i}$ by the above big commutative diagram. By Nakayama's lemma, Coker $b_{i}$ is zero on a neighborhood of $F_{k}$ in $F_{i}$. This implies that $\mathcal{J}\left(\left.\varphi\right|_{F_{i}}\right)=$ $\left.\mathcal{J}(\varphi)\right|_{F_{i}}$ on a neighborhood of $F_{k}$ in $F_{i}$. Thus, we obtain that $\left.\mathcal{J}\left(\left.\varphi\right|_{F_{i-1}}\right)\right|_{F_{i}}=$ $\left.\mathcal{J}(\varphi)\right|_{F_{i}}$ on a neighborhood of $F_{k}$ in $F_{i}$ since we have $\left.\mathcal{J}\left(\left.\varphi\right|_{F_{i}}\right) \subset \mathcal{J}\left(\left.\varphi\right|_{F_{i-1}}\right)\right|_{F_{i}} \subset$ $\left.\mathcal{J}(\varphi)\right|_{F_{i}}$ by Lemma3.1. By repeating this argument, we see that $\mathcal{J}\left(\left.\varphi\right|_{F_{j}}\right)=\left.\mathcal{J}(\varphi)\right|_{F_{j}}$ on a neighborhood of $F_{k}$ in $F_{j}$ for every $j$. This is the desired property.

Lemma 3.4. Assume that $\left\{H_{1}, \cdots, H_{m}\right\}$ satisfies condition in Lemma 3.2. Let $H_{m+1}$ be a smooth Cartier divisor on $X$ such that $\sum_{i=1}^{m+1} H_{i}$ is a simple normal crossing divisor on $X$ and that $H_{m+1}$ contains no associated primes of

$$
\mathcal{O}_{X} / \mathcal{J}(\varphi) \quad \text { and } \quad \mathcal{O}_{H_{i_{1}} \cap \cdots \cap H_{i_{l}}} /\left.\mathcal{J}(\varphi)\right|_{H_{i_{1}} \cap \cdots \cap H_{i_{l}}}
$$

for every $1 \leq i_{1}<\cdots<i_{l} \leq m$. Then $\left\{H_{1}, \cdots, H_{m}, H_{m+1}\right\}$ also satisfies condition

Proof. This is obvious from Remark 3.3 (3).

Lemma 3.5. Let $\Lambda_{0}$ be a sublinear system of a free linear system $\Lambda$ on $X$ with $\operatorname{dim} \Lambda_{0} \geq 1$. Assume that $\left\{H_{1}, \cdots, H_{m}\right\}$ satisfies condition in Lemma 3.2 . We put

$$
\mathcal{F}_{0}:=\left\{D \in \Lambda_{0} \mid\left\{H_{1}, \cdots, H_{m}, D\right\} \text { satisfies } \mathbf{\Lambda}\right\} .
$$

Then $\mathcal{F}_{0}$ is Zariski open in $\Lambda_{0}$. In particular, if $\mathcal{F}_{0}$ is not empty, then it is a dense Zariski open set of $\Lambda_{0}$.

Moreover, we assume that there exists $D_{0} \in \mathcal{F}_{0}$ such that $\mathcal{J}\left(\left.\varphi\right|_{V}\right)=\left.\mathcal{J}(\varphi)\right|_{V}$, where $V$ is an irreducible component of $H_{1} \cap \cdots \cap H_{m} \cap D_{0}$. Let $D$ be a member of $\mathcal{F}_{0}$ such that $V$ is an irreducible component of $H_{1} \cap \cdots \cap H_{m} \cap D$. Then $\mathcal{J}\left(\left.\varphi\right|_{D}\right)=$ $\left.\mathcal{J}(\varphi)\right|_{D}$ holds on a neighborhood of $V$ in $D$.

Proof. We put

$$
\mathcal{F}:=\left\{D \in \Lambda \mid\left\{H_{1}, \cdots, H_{m}, D\right\} \text { satisfies } \mathbf{\$}\right\} .
$$

Then, by Remark $3.3(3)$ and Lemma 3.4, it is easy to see that $\mathcal{F}$ is a dense Zariski open set in $\Lambda$ since $\Lambda$ is a free linear system on $X$. Therefore $\mathcal{F}_{0}=\mathcal{F} \cap \Lambda_{0}$ is Zariski open in $\Lambda_{0}$. By Lemma 3.2 the equality $\mathcal{J}\left(\left.\varphi\right|_{D}\right)=\left.\mathcal{J}(\varphi)\right|_{D}$ holds on a neighborhood of $V$ in $D$ if $D \in \mathcal{F}_{0}$ and $V$ is an irreducible component of $H_{1} \cap \cdots \cap H_{m} \cap D$. We note that we do not need the compactness of $X$ in the proof of Lemma 3.2 Therefore, we can shrink $X$ and assume that $V=H_{1} \cap \cdots \cap H_{m} \cap D$ in the above argument.

The following theorem (see Theorem 1.10) is one of the key results of this paper.

Theorem 3.6 (Density of good divisors: Theorem 1.10). Let $X$ be a compact complex manifold, let $\Lambda$ be a free linear system on $X$ with $\operatorname{dim} \Lambda \geq 1$, and let $\varphi$ be a quasi-plurisubharmonic function on $X$. We put

$$
\mathcal{G}:=\left\{H \in \Lambda \mid H \text { is smooth and } \mathcal{J}\left(\left.\varphi\right|_{H}\right)=\left.\mathcal{J}(\varphi)\right|_{H}\right\} .
$$

Then $\mathcal{G}$ is dense in $\Lambda$ in the classical topology.

Proof. We may assume that $\varphi \not \equiv-\infty$. Throughout this proof, we put $f:=\Phi_{\Lambda}$ : $X \rightarrow Y:=f(X) \subset \mathbb{P}^{N}$. Note that $N=\operatorname{dim} \Lambda$. We divide the proof into four steps. 
Step 0 (Idea of the proof). In this step, we will explain an idea of the proof.

A general member $H$ of $\Lambda$ is smooth by Bertini's theorem, and it always satisfies that $\left.\mathcal{J}\left(\left.\varphi\right|_{H}\right) \subset \mathcal{J}(\varphi)\right|_{H}$ by Lemma 3.1. Hence, the problem is to check that the opposite inclusion holds for any member of a dense subset in $\Lambda$.

If $\operatorname{dim} \Lambda=1$, that is, $\Lambda$ is a pencil, then a member $H$ of $\Lambda$ is a fiber of the morphism $f=\Phi_{\Lambda}: X \rightarrow \mathbb{P}^{1}$ at a point $P \in \mathbb{P}^{1}$. By Fubini's theorem, we have $\left.\mathcal{J}\left(\left.\varphi\right|_{f^{-1}(P)}\right) \supset \mathcal{J}(\varphi)\right|_{f^{-1}(P)}$ for almost all $P \in \mathbb{P}^{1}$. This is the desired statement when $\operatorname{dim} \Lambda=1$. In general, we have $H_{1} \cap H_{2} \neq \emptyset$ for two general members $H_{1}$ and $H_{2}$ of $\Lambda$. For this reason, we choose $H_{1}$ and $H_{2}$ suitably (see Step 2 and Step 3), take the blow-up $Z \rightarrow X$ along $H_{1} \cap H_{2}$, and reduce the problem to the pencil case (see Step 4).

Step 1. In this step, we will prove the theorem when $\operatorname{dim} Y=1$.

Let $\psi_{0}, \ldots, \psi_{N}$ be a basis of $H^{0}\left(\mathbb{P}^{N}, \mathcal{O}_{\mathbb{P}^{N}}(1)\right)$. We put

$$
\mathscr{Y}=\left\{\left(y,\left[a_{0}: \cdots: a_{N}\right]\right) \in Y \times \mathbb{P}^{N} \mid a_{0} \psi_{0}(y)+\cdots+a_{N} \psi_{N}(y)=0\right\} \subset Y \times \mathbb{P}^{N}
$$

and consider the following commutative diagram:

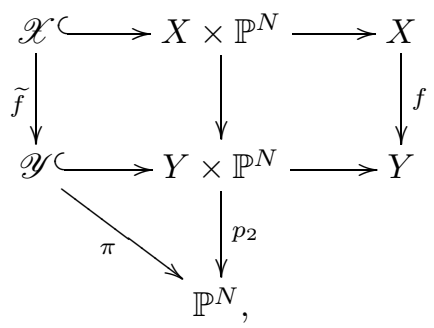

where $\mathscr{X} \hookrightarrow X \times \mathbb{P}^{N} \rightarrow X$ is the base change of $\mathscr{Y} \hookrightarrow Y \times \mathbb{P}^{N} \rightarrow Y$ by $f: X \rightarrow Y$, $p_{2}$ is the second projection, and $\pi=p_{2} \mid \mathscr{y}$. We can easily see that there exists a nonempty Zariski open set $U$ of $\mathbb{P}^{N}$ such that $\pi$ and $\tilde{f}$ are étale and smooth over $U$, respectively. We note that $\Lambda=f^{*}\left|\mathcal{O}_{\mathbb{P}^{N}}(1)\right|$ by construction. Let $H$ be a member of $\Lambda$ corresponding to a point of $U$. Then $H$ is smooth and $\left.\mathcal{J}\left(\left.\varphi\right|_{H}\right) \subset \mathcal{J}(\varphi)\right|_{H}$ holds by Lemma 3.1. On the other hand, by applying Fubini's theorem to $(\pi \circ \widetilde{f})^{-1}(U) \rightarrow U$, the opposite inclusion $\left.\mathcal{J}(\varphi)\right|_{H} \subset \mathcal{J}\left(\left.\varphi\right|_{H}\right)$ holds for almost all $H \in \Lambda$. This means that $\mathcal{G}$ is dense in $\Lambda$ in the classical topology.

Step 2. In this step, we will prove the following preparatory lemma.

Lemma 3.7. Let $D_{1}$ and $D_{2}$ be two members of $\Lambda$ such that $\left\{D_{1}, D_{2}\right\}$ satisfies condition in Lemma 3.2. Let $\mathcal{P}_{0}$ be the pencil spanned by $D_{1}$ and $D_{2}$. Then, for almost all $D \in \mathcal{P}_{0}$, the member $D$ is smooth, $\{D\}$ satisfies condition $\mathbf{\phi}$, and $\mathcal{J}\left(\left.\varphi\right|_{D}\right)=\left.\mathcal{J}(\varphi)\right|_{D}$ holds outside $D_{1} \cap D_{2}$.

Proof of Lemma 3.7. Let $A_{i}$ be a hyperplane in $\mathbb{P}^{N}$ such that $D_{i}=f^{*} A_{i}$, and pr: $\mathbb{P}^{N} \rightarrow \mathbb{P}^{1}$ be the linear projection from the subspace $A_{1} \cap A_{2} \cong \mathbb{P}^{N-2}$. Then the meromorphic map $X \rightarrow \mathbb{P}^{1}$ associated with $\mathcal{P}_{0}$ is the composition of $f: X \rightarrow \mathbb{P}^{N}$ and pr: $\mathbb{P}^{N} \rightarrow \mathbb{P}^{1}$. Since the blow-up of $\mathbb{P}^{N}$ along $A_{1} \cap A_{2}$ gives an elimination of the indeterminacy locus of pr: $\mathbb{P}^{N} \rightarrow \mathbb{P}^{1}$, the blow-up $p: Z \rightarrow X$ along $D_{1} \cap D_{2}$ 
satisfies the following commutative diagram:

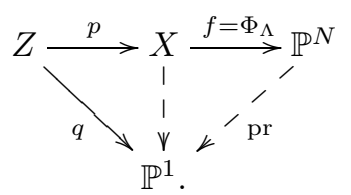

By applying Fubini's theorem to $q: Z \rightarrow \mathbb{P}^{1}$, we obtain that $\mathcal{J}\left(\left.p^{*} \varphi\right|_{q^{-1}(Q)}\right)=$ $\left.\mathcal{J}\left(p^{*} \varphi\right)\right|_{q^{-1}(Q)}$ for almost all $Q \in \mathbb{P}^{1}$. Lemma 3.5 implies that $\{D\}$ satisfies condition \$ for almost all $D \in \mathcal{P}_{0}$. The desired properties follow since $p$ is an isomorphism outside $D_{1} \cap D_{2}$.

Step 3. In this step, we will find a smooth member $H$ of $\Lambda$ such that $\mathcal{J}\left(\left.\varphi\right|_{H}\right)=$ $\left.\mathcal{J}(\varphi)\right|_{H}$ and that $\{H\}$ satisfies condition

From now on, we assume that $\operatorname{dim} \Lambda \geq 2$ and that the statement of Theorem 3.6 holds for lower dimensional free linear systems. We put $l:=\operatorname{dim} Y$. By Step 11 we have a smooth member $H$ of $\Lambda$ with the desired properties when $l=1$. Therefore, we may assume that $l \geq 2$. We take two general hyperplanes $B_{1}$ and $B_{2}$ of $\mathbb{P}^{N}$. We put $D_{1}:=f^{*} B_{1}$ and $D_{2}:=f^{*} B_{2}$. By Lemma 3.7, we can take a hyperplane $A_{1}$ of $\mathbb{P}^{N}$ such that $X_{1}:=f^{*} A_{1}$ is smooth, $\left\{X_{1}\right\}$ satisfies condition \$, and $\mathcal{J}\left(\left.\varphi\right|_{X_{1}}\right)=\left.\mathcal{J}(\varphi)\right|_{X_{1}}$ outside $D_{1} \cap D_{2}$. Let $\left.\Lambda\right|_{X_{1}}$ be the linear system on $X_{1}$ defined by $f_{1}: X_{1}=X \cap f^{-1}\left(A_{1}\right) \rightarrow Y \cap A_{1} \subset A_{1} \cong \mathbb{P}^{N-1}$, that is, the set of pull-backs of the hyperplanes in $A_{1} \cong \mathbb{P}^{N-1}$ by $f_{1}$. By construction, we have $\left.\operatorname{dim} \Lambda\right|_{X_{1}}=\operatorname{dim} \Lambda-1$. Thus, we see that

$$
\left\{H \in \Lambda \mid X_{1} \cap H \text { is smooth and } \mathcal{J}\left(\left.\varphi\right|_{X_{1} \cap H}\right)=\left.\mathcal{J}\left(\left.\varphi\right|_{X_{1}}\right)\right|_{X_{1} \cap H}\right\}
$$

is dense in $\Lambda$ in the classical topology by the induction hypothesis. Then we can take general hyperplanes $A_{2}, A_{3}, \cdots, A_{l}$ of $\mathbb{P}^{N}$ such that $\operatorname{dim}\left(A_{1} \cap \cdots \cap A_{l} \cap Y\right)=0$ and that $f^{-1}(Q)$ is smooth and

$$
\mathcal{J}\left(\left.\varphi\right|_{f^{-1}(Q)}\right)=\left.\mathcal{J}\left(\left.\varphi\right|_{X_{1}}\right)\right|_{f^{-1}(Q)}
$$

for every $Q \in A_{1} \cap \cdots \cap A_{l} \cap Y$ by using the induction hypothesis repeatedly. Without loss of generality, we may assume that $f^{-1}(Q) \cap D_{1} \cap D_{2}=\emptyset$ for every $Q \in A_{1} \cap \cdots \cap A_{l} \cap Y$. Since

$$
\mathcal{J}\left(\left.\varphi\right|_{X_{1}}\right)=\left.\mathcal{J}(\varphi)\right|_{X_{1}}
$$

holds outside $D_{1} \cap D_{2}$,

$$
\left.\mathcal{J}\left(\left.\varphi\right|_{X_{1}}\right)\right|_{f^{-1}(Q)}=\left.\mathcal{J}(\varphi)\right|_{f^{-1}(Q)}
$$

holds for every $Q \in A_{1} \cap \cdots \cap A_{l} \cap Y$. Therefore, we have

$$
\mathcal{J}\left(\left.\varphi\right|_{f^{-1}(Q)}\right)=\left.\mathcal{J}\left(\left.\varphi\right|_{X_{1}}\right)\right|_{f^{-1}(Q)}=\left.\mathcal{J}(\varphi)\right|_{f^{-1}(Q)}
$$

for every $Q \in A_{1} \cap \cdots \cap A_{l} \cap Y$ by (3.5) and (3.6). We may assume that $\left\{X_{1}=\right.$ $\left.f^{*} A_{1}, f^{*} A_{2}, \cdots, f^{*} A_{l}\right\}$ satisfies condition $\boldsymbol{\phi}$. We take one point $P$ of $A_{1} \cap \cdots \cap A_{l} \cap Y$ and fix $A_{2}, \cdots, A_{l}$. By applying Lemma 3.5 to the linear system

$$
\Lambda_{0}:=\left\{D \in \Lambda \mid f^{-1}(P) \subset D\right\},
$$

we see that

$$
\mathcal{F}_{0}:=\left\{D \in \Lambda_{0} \mid\left\{D, f^{*} A_{2}, \cdots, f^{*} A_{l}\right\}\right. \text { satisfies }
$$


is Zariski open in $\Lambda_{0}$. Note that $\mathcal{F}_{0}$ is nonempty by $X_{1}=f^{*} A_{1} \in \mathcal{F}_{0}$. By the latter conclusion of Lemma 3.5, we have:

Lemma 3.8. Let $A_{g}$ be a general hyperplane of $\mathbb{P}^{N}$ passing through $P$. We put $X_{g}:=f^{*} A_{g}$. Then $\mathcal{J}\left(\left.\varphi\right|_{X_{g}}\right)=\left.\mathcal{J}(\varphi)\right|_{X_{g}}$ holds on a neighborhood of $f^{-1}(P)$ in $X_{g}$.

Let $\pi: X^{\prime} \rightarrow X$ be the blow-up along $f^{-1}(P)$ and let $\mathrm{Bl}_{P}\left(\mathbb{P}^{N}\right) \rightarrow \mathbb{P}^{N}$ be the blowup of $\mathbb{P}^{N}$ at $P$. The induced morphism $\alpha: X^{\prime} \rightarrow \mathrm{Bl}_{P}\left(\mathbb{P}^{N}\right)$ and the linear projection $\gamma: \mathbb{P}^{N} \rightarrow \mathbb{P}^{N-1}$ from $P \in \mathbb{P}^{N}$ satisfy the following commutative diagram.

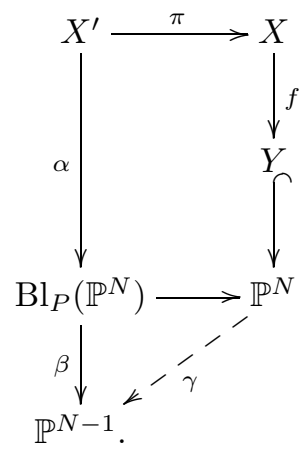

We put $f^{\prime}:=\beta \circ \alpha$ and $Y^{\prime}:=f^{\prime}\left(X^{\prime}\right)$. By applying the induction hypothesis to $f^{\prime}: X^{\prime} \rightarrow Y^{\prime} \subset \mathbb{P}^{N-1}$, we can take a general hyperplane $A$ of $\mathbb{P}^{N-1}$ such that $f^{\prime *} A$ is smooth and that

$$
\mathcal{J}\left(\left.\pi^{*} \varphi\right|_{f^{\prime-1}(A)}\right)=\left.\mathcal{J}\left(\pi^{*} \varphi\right)\right|_{f^{\prime-1}(A)} .
$$

Let $A_{0}$ be the hyperplane of $\mathbb{P}^{N}$ spanned by $P$ and $A$. Then we can see that

$$
\left\{f^{*} A_{2}, \cdots, f^{*} A_{l}, H:=f^{*} A_{0}\right\}
$$

satisfies condition since $A$ is a general hyperplane of $\mathbb{P}^{N-1}$. We see that $\mathcal{J}\left(\left.\varphi\right|_{H}\right)=$ $\left.\mathcal{J}(\varphi)\right|_{H}$ by (3.7) and Lemma 3.8, and that $\{H\}$ satisfies condition $\boldsymbol{\phi}$ by (3.8). Therefore this $H$ has the desired properties.

Step 4. In this final step, we will prove that $\mathcal{G}$ is dense in $\Lambda$ in the classical topology.

We will use the induction on $\operatorname{dim} X$. If $\operatorname{dim} X=1$, then $\operatorname{dim} Y=1$. Therefore, by Step 1 we see that $\mathcal{G}$ is dense in $\Lambda$ in the classical topology. Therefore, we assume that $\operatorname{dim} X \geq 2$. If $\operatorname{dim} Y=1$, then $\mathcal{G}$ is dense by Step 1] Thus, we may assume that $\operatorname{dim} \Lambda \geq \operatorname{dim} Y \geq 2$. By Step 3, we can take a smooth member $H_{0}$ of $\Lambda$ such that $\mathcal{J}\left(\left.\varphi\right|_{H_{0}}\right)=\left.\mathcal{J}(\varphi)\right|_{H_{0}}$ and that $\left\{H_{0}\right\}$ satisfies condition $\mathbf{\$}$. By applying the induction hypothesis to $\left.\Lambda\right|_{H_{0}}$, we see that

$$
\mathcal{G}^{\prime}:=\left\{H^{\prime} \in \Lambda \mid H_{0} \cap H^{\prime} \text { is smooth and } \mathcal{J}\left(\left.\varphi\right|_{H_{0} \cap H^{\prime}}\right)=\left.\mathcal{J}\left(\left.\varphi\right|_{H_{0}}\right)\right|_{H_{0} \cap H^{\prime}}\right\}
$$

is dense in $\Lambda$ in the classical topology. Since $\Lambda$ is a free linear system, we know that

$$
\left\{H^{\prime} \in \Lambda \mid\left\{H_{0}, H^{\prime}\right\} \text { satisfies } \boldsymbol{\phi}\right\}
$$

is a nonempty Zariski open set in $\Lambda$. Therefore,

$$
\mathcal{G}^{\prime \prime}:=\left\{H^{\prime} \in \mathcal{G}^{\prime} \mid\left\{H_{0}, H^{\prime}\right\}\right. \text { satisfies }
$$

is also dense in $\Lambda$ in the classical topology. We note that

$$
\mathcal{J}\left(\left.\varphi\right|_{H_{0} \cap H^{\prime}}\right)=\left.\mathcal{J}\left(\left.\varphi\right|_{H_{0}}\right)\right|_{H_{0} \cap H^{\prime}}=\left.\mathcal{J}(\varphi)\right|_{H_{0} \cap H^{\prime}}
$$


for every $H^{\prime} \in \mathcal{G}^{\prime}$ since $\mathcal{J}\left(\left.\varphi\right|_{H_{0}}\right)=\left.\mathcal{J}(\varphi)\right|_{H_{0}}$. Therefore, we obtain that

$$
\mathcal{J}\left(\left.\varphi\right|_{H_{0} \cap H^{\prime}}\right)=\left.\mathcal{J}\left(\left.\varphi\right|_{H^{\prime}}\right)\right|_{H_{0} \cap H^{\prime}}=\left.\mathcal{J}(\varphi)\right|_{H_{0} \cap H^{\prime}}
$$

for every $H^{\prime} \in \mathcal{G}^{\prime \prime}$. By the latter conclusion of Lemma 3.5, (3.9) indicates that $\mathcal{J}\left(\left.\varphi\right|_{H^{\prime}}\right)=\left.\mathcal{J}(\varphi)\right|_{H^{\prime}}$ on a neighborhood of $H_{0} \cap H^{\prime}$ in $H^{\prime}$ for every $H^{\prime} \in \mathcal{G}^{\prime \prime}$. We consider the pencil $\mathcal{P}_{H^{\prime}}$ spanned by $H_{0}$ and $H^{\prime} \in \mathcal{G}^{\prime \prime}$, that is, the sublinear system of $\Lambda$ spanned by $H_{0}$ and $H^{\prime}$. Let $D$ be a general member of $\mathcal{P}_{H^{\prime}}$. Then, by Lemma 3.5. $\left\{H_{0}, D\right\}$ satisfies and $\mathcal{J}\left(\left.\varphi\right|_{D}\right)=\left.\mathcal{J}(\varphi)\right|_{D}$ holds on a neighborhood of $H_{0} \cap H^{\prime}$ in $D$. Hence, by Lemma 3.7, we say that almost all members of $\mathcal{P}_{H^{\prime}}$ are contained in $\mathcal{G}$. By this observation, we obtain that $\mathcal{G}$ is dense in $\Lambda$ in the classical topology.

Thus, we obtain the desired statement.

The following examples show that $\mathcal{G}$ in Theorem 1.10 (Theorem 3.6) is not always Zariski open in $\Lambda$, or even an intersection of countably many nonempty Zariski open sets of $\Lambda$

Example 3.9. We put

$$
\psi(z):=\sum_{k=1}^{\infty} 2^{-k} \log \left|z-\frac{1}{k}\right|
$$

for $z \in \mathbb{C}$. Then it is easy to see that $\psi(z)$ is smooth for $|z| \geq 2$. By using a suitable partition of unity, we can construct a function $\varphi(z)$ on $\mathbb{P}^{1}$ such that $\varphi(z)=\psi(z)$ for $|z| \leq 3$ and that $\varphi(z)$ is smooth for $|z| \geq 2$ on $\mathbb{P}^{1}$. We can see that $\varphi$ is a quasi-plurisubharmonic function on $\mathbb{P}^{1}$. Since the Lelong number $\nu(\varphi, 1 / n)$ of $\varphi$ at $1 / n$ is $2^{-n}$ for every positive integer $n$, we see that $\mathcal{J}(\varphi)=\mathcal{O}_{\mathbb{P}^{1}}$ by Skoda's theorem (see, for example, [D3, (5.6) Lemma]). Therefore $\left.\mathcal{J}(\varphi)\right|_{P}=\mathcal{O}_{P}$ for every $P \in \mathbb{P}^{1}$. On the other hand, we have $\varphi(1 / n)=-\infty$ for every positive integer $n$. If $P=1 / n$ for some positive integer $n$, then $\mathcal{J}\left(\left.\varphi\right|_{P}\right)=0$. Thus

$$
\mathcal{G}:=\left\{H \in\left|\mathcal{O}_{\mathbb{P}^{1}}(1)\right|\left|\mathcal{J}\left(\left.\varphi\right|_{H}\right)=\mathcal{J}(\varphi)\right|_{H}\right\}
$$

is not a Zariski open set of $\left|\mathcal{O}_{\mathbb{P}^{1}}(1)\right|\left(\simeq \mathbb{P}^{1}\right)$.

Example 3.10. We put $K:=\{z \in \mathbb{C}|| z \mid \leq 1\}$. Let $\left\{w_{n}\right\}_{n=1}^{\infty}$ be a countable dense subset of $K$ and let $\left\{a_{n}\right\}_{n=1}^{\infty}$ be positive real numbers such that $\sum_{n=1}^{\infty} a_{n}<\infty$. We put

$$
\psi(z):=\sum_{n=1}^{\infty} a_{n} \log \left|z-w_{n}\right|
$$

for $z \in \mathbb{C}$. Then we see that

- $\psi$ is subharmonic on $\mathbb{C}$ and $\psi \not \equiv-\infty$,

- $\psi=-\infty$ on an uncountable dense subset of $K$, and

- $\psi$ is discontinuous almost everywhere on $K$.

For the details, see [Ra, Theorem 2.5.4]. By using a suitable partition of unity, we can construct a function $\varphi(z)$ on $\mathbb{P}^{1}$ such that $\varphi(z)=\psi(z)$ for $|z| \leq 3$ and that $\varphi(z)$ is smooth for $|z| \geq 2$ on $\mathbb{P}^{1}$. Then we can see that $\varphi$ is a quasi-plurisubharmonic function on $\mathbb{P}^{1}$. In this case,

$$
\mathcal{G}:=\left\{H \in\left|\mathcal{O}_{\mathbb{P}^{1}}(1)\right|\left|\mathcal{J}\left(\left.\varphi\right|_{H}\right)=\mathcal{J}(\varphi)\right|_{H}\right\}
$$

cannot be written as an intersection of countably many nonempty Zariski open sets of $\left|\mathcal{O}_{\mathbb{P}^{1}}(1)\right|$. 
As a direct consequence of Theorem 3.6, we have:

Corollary 3.11 (Generic restriction theorem). Let $X$ be a compact complex manifold and let $\varphi$ be a quasi-plurisubharmonic function on $X$. Let $\Lambda$ be a free linear system on $X$ with $\operatorname{dim} \Lambda \geq 1$. We put

$$
\mathcal{H}:=\left\{H \in \mathcal{G} \mid H \text { contains no associated primes of } \mathcal{O}_{X} / \mathcal{J}(\varphi)\right\},
$$

where

$$
\mathcal{G}:=\left\{H \in \Lambda \mid H \text { is smooth and } \mathcal{J}\left(\left.\varphi\right|_{H}\right)=\left.\mathcal{J}(\varphi)\right|_{H}\right\}
$$

as in Theorem 3.6. Then $\mathcal{H}$ is dense in $\Lambda$ in the classical topology. Moreover, the following short sequence

$$
0 \rightarrow \mathcal{J}(\varphi) \otimes \mathcal{O}_{X}(-H) \rightarrow \mathcal{J}(\varphi) \rightarrow \mathcal{J}\left(\left.\varphi\right|_{H}\right) \rightarrow 0
$$

is exact for any member $H$ of $\mathcal{H}$.

Proof. It is easy to see that

$$
\left\{H \in \Lambda \mid H \text { contains no associated primes of } \mathcal{O}_{X} / \mathcal{J}(\varphi)\right\}
$$

is a nonempty Zariski open set of $\Lambda$ since $\Lambda$ is a free linear system on $X$. Therefore $\mathcal{H}$ is dense in $\Lambda$ in the classical topology by Theorem 3.6 (see Theorem 1.10).

Let $H$ be a member of $\mathcal{H}$. Then we obtain the following commutative diagram (see also (3.2)):

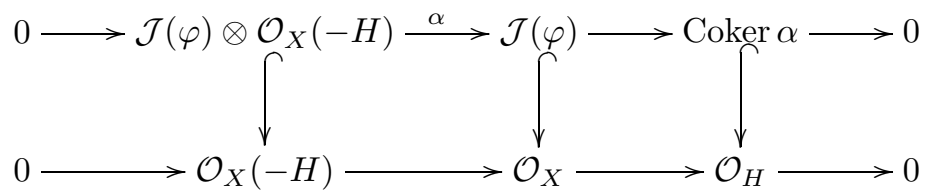

As in the proof of Lemma 3.2 we obtain Coker $\alpha=\left.\mathcal{J}(\varphi)\right|_{H}$. Since $H \in \mathcal{H} \subset \mathcal{G}$, we have $\left.\mathcal{J}(\varphi)\right|_{H}=\mathcal{J}\left(\left.\varphi\right|_{H}\right)$. Therefore, we obtain the desired short exact sequence (3.10).

We will use Corollary 3.11 in Step 3 in the proof of Proposition 1.9 (see Section (4). We close this section with a remark on the multiplier ideal sheaves associated with effective $\mathbb{Q}$-divisors on smooth projective varieties.

Remark 3.12 (Multiplier ideal sheaves for effective $\mathbb{Q}$-divisors). Let $X$ be a smooth projective variety and let $D$ be an effective $\mathbb{Q}$-divisor on $X$. Let $S$ be a smooth hypersurface in $X$. We assume that $S$ is not contained in any component of $D$. Then we obtain the following short exact sequence:

$$
0 \rightarrow \mathcal{J}(X, D) \otimes \mathcal{O}_{X}(-S) \rightarrow \operatorname{Adj}_{S}(X, D) \rightarrow \mathcal{J}\left(S,\left.D\right|_{S}\right) \rightarrow 0,
$$

where $\mathcal{J}(X, D)$ (resp. $\mathcal{J}\left(S,\left.D\right|_{S}\right)$ ) is the multiplier ideal sheaf associated with $D$ (resp. $\left.D\right|_{S}$ ). Note that $\operatorname{Adj}_{S}(X, D)$ is the adjoint ideal of $D$ along $S$ (see, for example, [L3, Theorem 3.3]). If $S$ is in general position with respect to $D$, then we can easily see that $\operatorname{Adj}_{S}(X, D)$ coincides with $\mathcal{J}(X, D)$. Let $H$ be a general member of a free linear system $\Lambda$ with $\operatorname{dim} \Lambda \geq 1$. Then we can easily see that

$$
\mathcal{J}\left(H,\left.D\right|_{H}\right)=\left.\mathcal{J}(X, D)\right|_{H}
$$

holds by the definition of the multiplier ideal sheaves for effective $\mathbb{Q}$-divisors (see, for example, [L2, Example 9.5.9]). 
By this observation, if $X$ is a smooth projective variety and $\varphi$ is a quasiplurisubharmonic function associated with an effective $\mathbb{Q}$-divisor $D$ on $X$, then $\mathcal{G}$ in Theorem [3.6 (see Theorem 1.10) and $\mathcal{H}$ in Corollary 3.11] are dense Zariski open in $\Lambda$ by (3.12). Moreover, we can easily check that (3.10) in Corollary 3.11 holds for general members $H$ of $\Lambda$ by (3.11).

\section{Proof of Proposition 1.9}

In this section, we prove Proposition 1.9 and explain how to reduce Corollary 1.7 and Theorem 1.4 to Theorem $\mathrm{D}$ and Theorem $\mathrm{A}$, respectively.

Proof of Proposition 1.9. Our proof of Proposition 1.9 consists of the following six steps:

Step 1 (Theorem $\mathrm{A} \Longrightarrow$ Theorem $\mathrm{B}$ ). Since $N_{1}$ is semiample, we can take a smooth Hermitian metric $h_{1}$ on $N_{1}$ such that $\sqrt{-1} \Theta_{h_{1}}\left(N_{1}\right) \geq 0$. We put $h_{2}:=h_{1}^{b / a}$. Then

$$
\sqrt{-1}\left(\Theta_{h h_{1}}\left(F \otimes N_{1}\right)-t \Theta_{h_{2}}\left(N_{2}\right)\right) \geq 0
$$

for $0<t \ll 1$. It follows that $\mathcal{J}\left(h h_{1}\right)=\mathcal{J}(h)$ since $h_{1}$ is smooth. Therefore, by Theorem A, we obtain the injectivity in Theorem B.

Step 2 (Theorem $\mathrm{B} \Longrightarrow$ Theorem $\mathrm{C}$ ). We assume that $R^{i} f_{*}\left(K_{X} \otimes F \otimes \mathcal{J}(h)\right.$ ) has a torsion subsheaf. Then we can find a very ample line bundle $H$ on $Y$ and $0 \neq t \in H^{0}(Y, H)$ such that

$$
\alpha: R^{i} f_{*}\left(K_{X} \otimes F \otimes \mathcal{J}(h)\right) \rightarrow R^{i} f_{*}\left(K_{X} \otimes F \otimes \mathcal{J}(h)\right) \otimes H
$$

induced by $\otimes t$ is not injective. We take a sufficiently large positive integer $m$ such that $\operatorname{Ker} \alpha \otimes H^{\otimes m}$ is generated by global sections. Then we have $H^{0}(Y, \operatorname{Ker} \alpha \otimes$ $\left.H^{\otimes m}\right) \neq 0$. Without loss of generality, by making $m$ sufficiently large, we may further assume that

$$
H^{p}\left(Y, R^{q} f_{*}\left(K_{X} \otimes F \otimes \mathcal{J}(h)\right) \otimes H^{\otimes m}\right)=0
$$

and

$$
H^{p}\left(Y, R^{q} f_{*}\left(K_{X} \otimes F \otimes \mathcal{J}(h)\right) \otimes H^{\otimes m+1}\right)=0
$$

for every $p>0$ and $q$ by the Serre vanishing theorem. By construction,

$$
H^{0}\left(Y, R^{i} f_{*}\left(K_{X} \otimes F \otimes \mathcal{J}(h)\right) \otimes H^{\otimes m}\right) \rightarrow H^{0}\left(Y, R^{i} f_{*}\left(K_{X} \otimes F \otimes \mathcal{J}(h)\right) \otimes H^{\otimes m+1}\right)
$$

induced by $\alpha$ is not injective. Thus, by (4.1), (4.2), and (4.3), we see that

$$
H^{i}\left(X, K_{X} \otimes F \otimes \mathcal{J}(h) \otimes f^{*} H^{\otimes m}\right) \rightarrow H^{i}\left(X, K_{X} \otimes F \otimes \mathcal{J}(h) \otimes f^{*} H^{\otimes m+1}\right)
$$

induced by $\otimes f^{*} t$ is not injective. This contradicts Theorem $\mathrm{B}$. Therefore $R^{i} f_{*}\left(K_{X} \otimes\right.$ $F \otimes \mathcal{J}(h))$ is torsion-free.

Step 3 (Theorem $\mathrm{B} \Longrightarrow$ Theorem $\mathrm{D}$ ). We use the induction on $\operatorname{dim} Y$. If $\operatorname{dim} Y=0$, then the statement is obvious. We take a sufficiently large positive integer $m$ and a general divisor $B \in\left|H^{\otimes m}\right|$ such that $D:=f^{-1}(B)$ is smooth, contains no associated primes of $\mathcal{O}_{X} / \mathcal{J}(h)$, and satisfies $\mathcal{J}\left(\left.h\right|_{D}\right)=\left.\mathcal{J}(h)\right|_{D}$ by Theorem 3.6 (see Theorem 1.10) and Corollary 3.11, By the Serre vanishing theorem, we may further assume that

$$
H^{i}\left(Y, R^{j} f_{*}\left(K_{X} \otimes F \otimes \mathcal{J}(h) \otimes N\right) \otimes H^{\otimes m}\right)=0
$$


for every $i>0$ and $j$. By Corollary 3.11 and adjunction, we have the following short exact sequence:

$$
\begin{aligned}
0 & \rightarrow K_{X} \otimes F \otimes \mathcal{J}(h) \otimes N \rightarrow K_{X} \otimes F \otimes \mathcal{J}(h) \otimes N \otimes f^{*} H^{\otimes m} \\
& \left.\left.\rightarrow K_{D} \otimes F\right|_{D} \otimes \mathcal{J}\left(\left.h\right|_{D}\right) \otimes N\right|_{D} \rightarrow 0 .
\end{aligned}
$$

Since $B$ is a general member of $\left|H^{\otimes m}\right|$, we may assume that $B$ contains no associated primes of $R^{j} f_{*}\left(K_{X} \otimes F \otimes \mathcal{J}(h) \otimes N\right)$ for every $j$. Hence, by (4.5), we can obtain

$$
\begin{aligned}
0 & \rightarrow R^{j} f_{*}\left(K_{X} \otimes F \otimes \mathcal{J}(h) \otimes N\right) \rightarrow R^{j} f_{*}\left(K_{X} \otimes F \otimes \mathcal{J}(h) \otimes N\right) \otimes H^{\otimes m} \\
& \rightarrow R^{j} f_{*}\left(\left.\left.K_{D} \otimes F\right|_{D} \otimes \mathcal{J}\left(\left.h\right|_{D}\right) \otimes N\right|_{D}\right) \rightarrow 0
\end{aligned}
$$

for every $j$. By using the long exact sequence and the induction on $\operatorname{dim} Y$, we obtain

$$
H^{i}\left(Y, R^{j} f_{*}\left(K_{X} \otimes F \otimes \mathcal{J}(h) \otimes N\right)\right)=H^{i}\left(Y, R^{j} f_{*}\left(K_{X} \otimes F \otimes \mathcal{J}(h) \otimes N\right) \otimes H^{\otimes m}\right)
$$

for every $i \geq 2$ and $j$. Thus we have

$$
H^{i}\left(Y, R^{j} f_{*}\left(K_{X} \otimes F \otimes \mathcal{J}(h) \otimes N\right)\right)=0
$$

for every $i \geq 2$ and $j$ by (4.4). By Leray's spectral sequence, (4.4), and (4.6), we have the following commutative diagram:

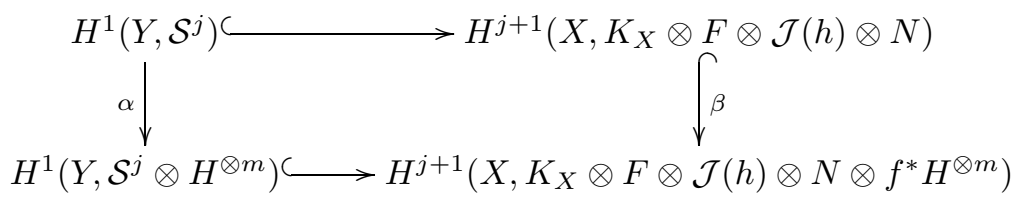

for every $j$, where $\mathcal{S}^{j}$ stands for $R^{j} f_{*}\left(K_{X} \otimes F \otimes \mathcal{J}(h) \otimes N\right)$. Since $\beta$ is injective by Theorem [B] we obtain that $\alpha$ is also injective. By (4.4), we have

$$
H^{1}\left(Y, R^{j} f_{*}\left(K_{X} \otimes F \otimes \mathcal{J}(h) \otimes N\right) \otimes H^{\otimes m}\right)=0
$$

for every $j$. Therefore, we have $H^{1}\left(Y, R^{j} f_{*}\left(K_{X} \otimes F \otimes \mathcal{J}(h) \otimes N\right)\right)=0$ for every $j$. Thus, we obtain the desired vanishing theorem in Theorem $\mathrm{D}$.

Step 4 (Theorems $\mathrm{C}$ and $\mathrm{D} \Longrightarrow$ Theorem $\mathrm{B}$ ). By replacing $s$ and $N_{2}$ with $s^{\otimes m}$ and $N_{2}^{\otimes m}$ for some positive integer $m$ (see also Remark 1.5), we may assume that $N_{2}$ is globally generated. We consider

$$
f:=\Phi_{\left|N_{2}\right|}: X \rightarrow Y .
$$

Then $N_{2} \simeq f^{*} H$ for some ample line bundle $H$ on $Y$ and $s=f^{*} t$ for some $t \in$ $H^{0}(Y, H)$. We take a smooth Hermitian metric $h_{1}$ on $N_{1}$ such that $\sqrt{-1} \Theta_{h_{1}}\left(N_{1}\right) \geq$ 0 . Then $\sqrt{-1} \Theta_{h h_{1}}\left(F \otimes N_{1}\right) \geq 0$ and $\mathcal{J}\left(h h_{1}\right)=\mathcal{J}(h)$. By Theorem Cl, we obtain that

$$
R^{i} f_{*}\left(K_{X} \otimes F \otimes \mathcal{J}(h) \otimes N_{1}\right)
$$

is torsion-free for every $i$. Therefore, the map

$$
R^{i} f_{*}\left(K_{X} \otimes F \otimes \mathcal{J}(h) \otimes N_{1}\right) \rightarrow R^{i} f_{*}\left(K_{X} \otimes F \otimes \mathcal{J}(h) \otimes N_{1}\right) \otimes H
$$

induced by $\otimes t$ is injective for every $i$. By $N_{2} \simeq f^{*} H$, we see that

$$
H^{0}\left(Y, R^{i} f_{*}\left(K_{X} \otimes F \otimes \mathcal{J}(h) \otimes N_{1}\right)\right) \rightarrow H^{0}\left(Y, R^{i} f_{*}\left(K_{X} \otimes F \otimes \mathcal{J}(h) \otimes N_{1} \otimes N_{2}\right)\right)
$$


induced by $\otimes t$ is injective for every $i$. By Theorem D, (4.7) implies that

$$
H^{i}\left(X, K_{X} \otimes F \otimes \mathcal{J}(h) \otimes N_{1}\right) \rightarrow H^{i}\left(X, K_{X} \otimes F \otimes \mathcal{J}(h) \otimes N_{1} \otimes N_{2}\right)
$$

induced by $\otimes s$ is injective for every $i$.

Step 5 (Theorem $\mathrm{D} \Longrightarrow$ Theorem $\mathrm{E}$ ). The following lemma implies that $R^{j} f_{*}\left(K_{X} \otimes\right.$ $F \otimes \mathcal{J}(h))$ is a GV-sheaf by [Sc, Theorem 25.5] (see also [Ha] and [PP]). For simplicity, we put $\mathcal{F}^{j}:=R^{j} f_{*}\left(K_{X} \otimes F \otimes \mathcal{J}(h)\right)$ for every $j$.

Lemma 4.1. For every finite étale morphism $p: B \rightarrow A$ of Abelian varieties and every ample line bundle $H$ on $B$, we have

$$
H^{i}\left(B, H \otimes p^{*} \mathcal{F}^{j}\right)=0
$$

for every $i>0$ and $j$.

Proof of Lemma 4.1. We put $Z:=B \times_{A} X$. Then we have the following commutative diagram:

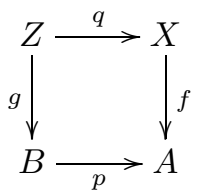

By construction, $q$ is also finite and étale. Therefore, we have $q^{*} K_{X}=K_{Z}$ and $q^{*} \mathcal{J}(h)=\mathcal{J}\left(q^{*} h\right)$. By the flat base change theorem,

$$
p^{*} R^{j} f_{*}\left(K_{X} \otimes F \otimes \mathcal{J}(h)\right) \simeq R^{j} g_{*}\left(K_{Z} \otimes q^{*} F \otimes \mathcal{J}\left(q^{*} h\right)\right) .
$$

By Theorem D, we obtain the desired vanishing (4.8).

Step 6 (Theorems $\mathrm{C}$ and $\mathrm{E} \Longrightarrow$ Theorem $\mathrm{E}$ ). By Theorem $\mathrm{C}$, we have $\mathcal{F}^{j}:=$ $R^{j} f_{*}\left(K_{X} \otimes F \otimes \mathcal{J}(h)\right)=0$ for $j>\operatorname{dim} X-\operatorname{dim} f(X)$. We consider the following spectral sequence:

$$
E_{2}^{p q}=H^{p}\left(A, \mathcal{F}^{q} \otimes L\right) \Rightarrow H^{p+q}\left(X, K_{X} \otimes F \otimes \mathcal{J}(h) \otimes f^{*} L\right)
$$

for every $L \in \operatorname{Pic}^{0}(A)$. Note that $\mathcal{F}^{j}$ is a GV-sheaf for every $j$ and that $\mathcal{F}^{j}=0$ for $j>\operatorname{dim} X-\operatorname{dim} f(X)$. Then we obtain

$$
\begin{aligned}
\operatorname{codim}_{\mathrm{Pic}^{0}(A)}\left\{L \in \operatorname{Pic}^{0}(A) \mid H^{i}\left(X, K_{X} \otimes F \otimes \mathcal{J}(h) \otimes f^{*} L\right)\right. & \neq 0\} \\
& \geq i-(\operatorname{dim} X-\operatorname{dim} f(X))
\end{aligned}
$$

for every $i \geq 0$.

We completed the proof of Proposition 1.9.

We prove Corollary 1.7 as an application of Theorem D. Proof of Corollary 1.7 (Theorem $\mathrm{D} \Longrightarrow$ Corollary 1.7). By Theorem D, we have

$$
H^{p}\left(Y, R^{i} f_{*}\left(K_{X} \otimes F \otimes \mathcal{J}(h)\right) \otimes H^{\otimes m-p}\right)=0
$$

for every $p \geq 1, i \geq 0$, and $m \geq \operatorname{dim} Y+1$. Thus, the Castelnuovo-Mumford regularity (see [L1, Section 1.8]) implies that $R^{i} f_{*}\left(K_{X} \otimes F \otimes \mathcal{J}(h)\right) \otimes H^{\otimes m}$ is globally generated for every $i \geq 0$ and $m \geq \operatorname{dim} Y+1$.

We close this section with a proof of Theorem 1.4 based on Theorem $\mathrm{A}$ for the reader's convenience. 
Proof of Theorem 1.4 ( Theorem $\mathrm{A} \Longrightarrow$ Theorem 1.4 ). Let $A$ be an ample line bundle on $V$. Then there exists a sufficiently large positive integer $m$ such that $A^{\otimes m}$ is very ample and that $H^{i}\left(V, K_{V} \otimes L \otimes \mathcal{J}\left(h_{L}\right) \otimes A^{\otimes m}\right)=0$ for every $i>0$ by the Serre vanishing theorem. We can take a smooth Hermitian metric $h_{A}$ on $A$ such that $\sqrt{-1} \Theta_{h_{A}}(A)$ is a smooth positive $(1,1)$-form on $V$. Therefore, we have $\sqrt{-1} \Theta_{h_{A}^{m}}\left(A^{\otimes m}\right) \geq 0$. By the condition $\sqrt{-1} \Theta_{h_{L}}(L) \geq \varepsilon \omega$, we see that $\sqrt{-1}\left(\Theta_{h_{L}}(L)-t \Theta_{h_{A}^{m}}\left(A^{\otimes m}\right)\right) \geq 0$ for some $0<t \ll 1$. We take a nonzero global section $s$ of $A^{\otimes m}$. By Theorem $\mathrm{A}$, we see that

$$
\times s: H^{i}\left(V, K_{V} \otimes L \otimes \mathcal{J}\left(h_{L}\right)\right) \rightarrow H^{i}\left(V, K_{V} \otimes L \otimes \mathcal{J}\left(h_{L}\right) \otimes A^{\otimes m}\right)
$$

is injective for every $i$. Thus, we obtain that $H^{i}\left(V, K_{V} \otimes L \otimes \mathcal{J}\left(h_{L}\right)\right)=0$ for every $i>0$.

\section{Proof of Theorem A}

In this section, we will give the proof of Theorem $\mathrm{A}$,

Theorem 5.1 (Theorem $\mathrm{A}$ ). Let $F$ (resp. $M$ ) be a line bundle on a compact Kähler manifold $X$ with a singular Hermitian metric $h$ (resp. a smooth Hermitian metric $\left.h_{M}\right)$ satisfying

$$
\sqrt{-1} \Theta_{h_{M}}(M) \geq 0 \text { and } \sqrt{-1} \Theta_{h}(F)-b \sqrt{-1} \Theta_{h_{M}}(M) \geq 0 \text { for some } b>0 .
$$

Then for a (nonzero) section $s \in H^{0}(X, M)$, the multiplication map induced by $\otimes s$

$$
\times s: H^{q}\left(X, K_{X} \otimes F \otimes \mathcal{J}(h)\right) \stackrel{\otimes s}{\longrightarrow} H^{q}\left(X, K_{X} \otimes F \otimes \mathcal{J}(h) \otimes M\right)
$$

is injective for every $q$. Here $K_{X}$ is the canonical bundle of $X$ and $\mathcal{J}(h)$ is the multiplier ideal sheaf of $h$.

Proof of Theorem 5.1 (Theorem $(\mathrm{A})$. The proof can be divided into four steps.

Step 1. Throughout the proof, we fix a Kähler form $\omega$ on $X$. For a given singular Hermitian metric $h$ on $F$, by applying DPS, Theorem 2.3] to the weight of $h$, we obtain a family of singular Hermitian metrics $\left\{h_{\varepsilon}\right\}_{1 \gg \varepsilon>0}$ on $F$ with the following properties:

(a) $h_{\varepsilon}$ is smooth on $Y_{\varepsilon}:=X \backslash Z_{\varepsilon}$, where $Z_{\varepsilon}$ is a proper closed analytic subset on $X$.

(b) $h_{\varepsilon^{\prime}} \leq h_{\varepsilon^{\prime \prime}} \leq h$ holds on $X$ when $\varepsilon^{\prime}>\varepsilon^{\prime \prime}>0$.

(c) $\mathcal{J}(h)=\mathcal{J}\left(h_{\varepsilon}\right)$ on $X$.

(d) $\sqrt{-1} \Theta_{h_{\varepsilon}}(F) \geq b \sqrt{-1} \Theta_{h_{M}}(M)-\varepsilon \omega$ on $X$.

Here property $(\mathrm{d})$ is obtained from the assumption $\sqrt{-1} \Theta_{h}(F) \geq b \sqrt{-1} \Theta_{h_{M}}(M)$.

The main difficulty of the proof is that $Z_{\varepsilon}$ may essentially depend on $\varepsilon$, compared to MaS4] in which $Z_{\varepsilon}$ is independent of $\varepsilon$. To overcome this difficulty, we consider suitable complete Kähler forms $\left\{\omega_{\varepsilon, \delta}\right\}_{\delta>0}$ on $Y_{\varepsilon}$ such that $\omega_{\varepsilon, \delta}$ converges to $\omega$ as $\delta \rightarrow 0$. To construct such complete Kähler forms, we first take a complete Kähler form $\omega_{\varepsilon}$ on $Y_{\varepsilon}$ with the following properties:

- $\omega_{\varepsilon}$ is a complete Kähler form on $Y_{\varepsilon}$.

- $\omega_{\varepsilon} \geq \omega$ on $Y_{\varepsilon}$.

- $\omega_{\varepsilon}=\sqrt{-1} \partial \bar{\partial} \Psi_{\varepsilon}$ for some bounded function $\Psi_{\varepsilon}$ on a neighborhood of every $p \in X$. 
See [F4, Section 3] for the construction of $\omega_{\varepsilon}$. For the Kähler form $\omega_{\varepsilon, \delta}$ on $Y_{\varepsilon}$ defined to be

$$
\omega_{\varepsilon, \delta}:=\omega+\delta \omega_{\varepsilon} \text { for } \varepsilon \text { and } \delta \text { with } 0<\delta \ll \varepsilon,
$$

it is easy to see the following properties hold:

(A) $\omega_{\varepsilon, \delta}$ is a complete Kähler form on $Y_{\varepsilon}=X \backslash Z_{\varepsilon}$ for every $\delta>0$.

(B) $\omega_{\varepsilon, \delta} \geq \omega$ on $Y_{\varepsilon}$ for every $\delta>0$.

(C) $\Psi+\delta \Psi_{\varepsilon}$ is a bounded local potential function of $\omega_{\varepsilon, \delta}$ and converges to $\Psi$ as $\delta \rightarrow 0$.

Here $\Psi$ is a local potential function of $\omega$. The first property enables us to consider harmonic forms on the noncompact $Y_{\varepsilon}$, and the third property enables us to construct the de Rham-Weil isomorphism from the $\bar{\partial}$-cohomology on $Y_{\varepsilon}$ to the Čech cohomology on $X$.

Remark 5.2. In the proof of Theorem [5.1 we actually consider only a countable sequence $\left\{\varepsilon_{k}\right\}_{k=1}^{\infty}$ (resp. $\left\{\delta_{\ell}\right\}_{\ell=1}^{\infty}$ ) conversing to zero since we need to apply Cantor's diagonal argument, but we often use the notation $\varepsilon$ (resp. $\delta$ ) for simplicity.

For the proof, it is sufficient to show that an arbitrary cohomology class $\eta \in$ $H^{q}\left(X, K_{X} \otimes F \otimes \mathcal{J}(h)\right)$ satisfying $s \eta=0 \in H^{q}\left(X, K_{X} \otimes F \otimes \mathcal{J}(h) \otimes M\right)$ is actually zero. We represent the cohomology class $\eta \in H^{q}\left(X, K_{X} \otimes F \otimes \mathcal{J}(h)\right)$ by a $\bar{\partial}$-closed $F$-valued $(n, q)$-form $u$ with $\|u\|_{h, \omega}<\infty$ by using the standard de Rham-Weil isomorphism

$$
H^{q}\left(X, K_{X} \otimes F \otimes \mathcal{J}(h)\right) \cong \frac{\operatorname{Ker} \bar{\partial}: L_{(2)}^{n, q}(F)_{h, \omega} \rightarrow L_{(2)}^{n, q+1}(F)_{h, \omega}}{\operatorname{Im} \bar{\partial}: L_{(2)}^{n, q-1}(F)_{h, \omega} \rightarrow L_{(2)}^{n, q}(F)_{h, \omega}} .
$$

Here $\bar{\partial}$ is the densely defined closed operator defined by the usual $\bar{\partial}$-operator and $L_{(2)}^{n, q}(F)_{h, \omega}$ is the $L^{2}$-space of $F$-valued $(n, q)$-forms on $X$ with respect to the $L^{2}$ norm $\|\bullet\|_{h, \omega}$ defined by

$$
\|\bullet\|_{h, \omega}^{2}:=\int_{X}|\bullet|_{h, \omega}^{2} d V_{\omega}
$$

where $d V_{\omega}:=\omega^{n} / n !$ and $n:=\operatorname{dim} X$. Our purpose is to prove that $u$ is $\bar{\partial}$-exact (namely, $\left.u \in \operatorname{Im} \bar{\partial} \subset L_{(2)}^{n, q}(F)_{h, \omega}\right)$ under the assumption that the cohomology class of $s u$ is zero in $H^{q}\left(X, K_{X} \otimes F \otimes \mathcal{J}(h) \otimes M\right)$.

From now on, we mainly consider the $L^{2}$-space $L_{(2)}^{n, q}\left(Y_{\varepsilon}, F\right)_{h_{\varepsilon}, \omega_{\varepsilon, \delta}}$ of $F$-valued $(n, q)$-forms on $Y_{\varepsilon}($ not $X)$ with respect to $h_{\varepsilon}$ and $\omega_{\varepsilon, \delta}$ (not $h$ and $\omega$ ). For simplicity we put

$$
L_{(2)}^{n, q}(F)_{\varepsilon, \delta}:=L_{(2)}^{n, q}\left(Y_{\varepsilon}, F\right)_{h_{\varepsilon}, \omega_{\varepsilon, \delta}} \quad \text { and } \quad\|\bullet\|_{\varepsilon, \delta}:=\|\bullet\|_{h_{\varepsilon}, \omega_{\varepsilon, \delta}} .
$$

The following inequality plays an important role in the proof:

$$
\|u\|_{\varepsilon, \delta} \leq\|u\|_{h, \omega_{\varepsilon, \delta}} \leq\|u\|_{h, \omega}<\infty .
$$

In particular, the norm $\|u\|_{\varepsilon, \delta}$ is uniformly bounded since the right-hand side is independent of $\varepsilon, \delta$. The first inequality follows from property (b) of $h_{\varepsilon}$, and the second inequality follows from Lemma 2.4 and property (B) of $\omega_{\varepsilon, \delta}$. Strictly speaking, the left-hand side should be $\left\|\left.u\right|_{Y_{\varepsilon}}\right\|_{\varepsilon, \delta}$, but we often omit the symbol of 
restriction. Now we have the following orthogonal decomposition (for example see [MaS4, Proposition 5.8]):

$$
L_{(2)}^{n, q}(F)_{\varepsilon, \delta}=\operatorname{Im} \bar{\partial} \oplus \mathcal{H}_{\varepsilon, \delta}^{n, q}(F) \oplus \operatorname{Im} \bar{\partial}_{\varepsilon, \delta}^{*} .
$$

Here $\bar{\partial}_{\varepsilon, \delta}^{*}$ is (the maximal extension of) the formal adjoint of the $\bar{\partial}$-operator and $\mathcal{H}_{\varepsilon, \delta}^{n, q}(F)$ is the set of harmonic $F$-valued $(n, q)$-forms on $Y_{\varepsilon}$, namely

$$
\mathcal{H}_{\varepsilon, \delta}^{n, q}(F):=\left\{w \in L_{(2)}^{n, q}(F)_{\varepsilon, \delta} \mid \bar{\partial} w=0 \text { and } \bar{\partial}_{\varepsilon, \delta}^{*} w=0\right\} .
$$

Remark 5.3. The formal adjoint coincides with the Hilbert space adjoint since $\omega_{\varepsilon, \delta}$ is complete for $\delta>0$ (see, for example, [D4, (3.2) Theorem in Chapter VIII]). The $\bar{\partial}$-operator also depends on $h_{\varepsilon}$ and $\omega_{\varepsilon, \delta}$ in the sense that the domain and range of the closed operator $\bar{\partial}$ depend on them, but we abbreviate $\bar{\partial}_{\varepsilon, \delta}$ to $\bar{\partial}$.

The $F$-valued $(n, q)$-form $u$ (representing $\eta$ ) belongs to $L_{(2)}^{n, q}(F)_{\varepsilon, \delta}$ by (5.1), and thus $u$ can be decomposed as follows:

$$
u=\bar{\partial} w_{\varepsilon, \delta}+u_{\varepsilon, \delta} \quad \text { for some } w_{\varepsilon, \delta} \in \operatorname{Dom} \bar{\partial} \subset L_{(2)}^{n, q-1}(F)_{\varepsilon, \delta} \text { and } u_{\varepsilon, \delta} \in \mathcal{H}_{\varepsilon, \delta}^{n, q}(F) .
$$

Note that the orthogonal projection of $u$ to $\operatorname{Im} \bar{\partial}_{\varepsilon, \delta}^{*}$ must be zero since $u$ is $\bar{\partial}$-closed.

Step 2. The purpose of this step is to prove Proposition 5.7, which reduces the proof to the study of the asymptotic behavior of the norm of $s u_{\varepsilon, \delta}$. When we consider a suitable limit of $u_{\varepsilon, \delta}$ in the following proposition, we need to carefully choose the $L^{2}$-space since the $L^{2}$-space $L_{(2)}^{n, q}(F)_{\varepsilon, \delta}$ depends on $\varepsilon$ and $\delta$. We remark that $\{\varepsilon\}_{\varepsilon>0}$ and $\{\delta\}_{\delta>0}$ denote countable sequences converging to zero (see Remark [5.2). Let $\left\{\delta_{0}\right\}_{\delta_{0}>0}$ denote another countable sequence converging to zero.

Proposition 5.4. There exist a subsequence $\left\{\delta_{\nu}\right\}_{\nu=1}^{\infty}$ of $\{\delta\}_{\delta>0}$ and $\alpha_{\varepsilon} \in L_{(2)}^{n, q}(F)_{h_{\varepsilon}, \omega}$ with the following properties:

- For any $\varepsilon, \delta_{0}>0$, as $\delta_{\nu}$ tends to 0 ,

$u_{\varepsilon, \delta_{\nu}}$ converges to $\alpha_{\varepsilon}$ with respect to the weak $L^{2}$-topology in $L_{(2)}^{n, q}(F)_{\varepsilon, \delta_{0}}$.

- For any $\varepsilon>0$,

$$
\left\|\alpha_{\varepsilon}\right\|_{h_{\varepsilon}, \omega} \leq \lim _{\delta_{0} \rightarrow 0}\left\|\alpha_{\varepsilon}\right\|_{\varepsilon, \delta_{0}} \leq \varliminf_{\delta_{\nu} \rightarrow 0}^{\lim }\left\|u_{\varepsilon, \delta_{\nu}}\right\|_{\varepsilon, \delta_{\nu}} \leq\|u\|_{h, \omega} .
$$

Remark 5.5. The weak limit $\alpha_{\varepsilon}$ does not depend on $\delta_{0}$, and the subsequence $\left\{\delta_{\nu}\right\}_{\nu=1}^{\infty}$ does not depend on $\varepsilon$ and $\delta_{0}$.

Proof of Proposition 5.4. For given $\varepsilon, \delta_{0}>0$, by taking a sufficiently small $\delta$ with $0<\delta<\delta_{0}$, we have

$$
\left\|u_{\varepsilon, \delta}\right\|_{\varepsilon, \delta_{0}} \leq\left\|u_{\varepsilon, \delta}\right\|_{\varepsilon, \delta} \leq\|u\|_{\varepsilon, \delta} \leq\|u\|_{h, \omega} .
$$

The first inequality follows from $\omega_{\varepsilon, \delta} \leq \omega_{\varepsilon, \delta_{0}}$ and Lemma 2.4 the second inequality follows since $u_{\varepsilon, \delta}$ is the orthogonal projection of $u$ with respect to $\varepsilon, \delta$, and the last inequality follows from (5.1). Since the right-hand side is independent of $\delta$, the family $\left\{u_{\varepsilon, \delta}\right\}_{\delta>0}$ is uniformly bounded in $L_{(2)}^{n, q}(F)_{\varepsilon, \delta_{0}}$. Therefore, there exists a subsequence $\left\{\delta_{\nu}\right\}_{\nu=1}^{\infty}$ of $\{\delta\}_{\delta>0}$ such that $u_{\varepsilon, \delta_{\nu}}$ converges to $\alpha_{\varepsilon, \delta_{0}}$ with respect to the weak $L^{2}$-topology in $L_{(2)}^{n, q}(F)_{\varepsilon, \delta_{0}}$ This subsequence $\left\{\delta_{\nu}\right\}_{\nu=1}^{\infty}$ may depend on 
$\varepsilon, \delta_{0}$, but we can choose a subsequence independent of them by applying Cantor's diagonal argument.

Now we show that $\alpha_{\varepsilon, \delta_{0}}$ does not depend on $\delta_{0}$. For arbitrary $\delta_{0}^{\prime}, \delta_{0}^{\prime \prime}$ with $0<$ $\delta_{0}^{\prime} \leq \delta_{0}^{\prime \prime}$, the natural inclusion $L_{(2)}^{n, q}(F)_{\varepsilon, \delta_{0}^{\prime}} \rightarrow L_{(2)}^{n, q}(F)_{\varepsilon, \delta_{0}^{\prime \prime}}$ is a bounded operator (continuous linear map) by $\|\bullet\|_{\varepsilon, \delta_{0}^{\prime \prime}} \leq\|\bullet\|_{\varepsilon, \delta_{0}^{\prime}}$, and thus $u_{\varepsilon, \delta_{\nu}}$ weakly converges to $\alpha_{\varepsilon, \delta_{0}^{\prime}}$ in not only $L_{(2)}^{n, q}(F)_{\varepsilon, \delta_{0}^{\prime}}$ but also $L_{(2)}^{n, q}(F)_{\varepsilon, \delta_{0}^{\prime \prime}}$ by Lemma 2.5. Therefore, it follows that $\alpha_{\varepsilon, \delta_{0}^{\prime}}=\alpha_{\varepsilon, \delta_{0}^{\prime \prime}}$ since the weak limit is unique.

Finally, we consider the norm of $\alpha_{\varepsilon}$. It is easy to see that

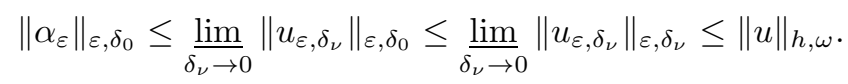

The first inequality follows since the norm is lower semicontinuous with respect to the weak convergence, the second inequality follows from $\omega_{\varepsilon, \delta_{0}} \geq \omega_{\varepsilon, \delta_{\nu}}$, and the last inequality follows from (5.3). Fatou's lemma yields

$$
\left\|\alpha_{\varepsilon}\right\|_{h_{\varepsilon}, \omega}^{2}=\int_{Y_{\varepsilon}}\left|\alpha_{\varepsilon}\right|_{h_{\varepsilon}, \omega}^{2} d V_{\omega} \leq \varliminf_{\delta_{0} \rightarrow 0}^{\lim } \int_{Y_{\varepsilon}}\left|\alpha_{\varepsilon}\right|_{h_{\varepsilon}, \omega_{\varepsilon}, \delta_{0}}^{2} d V_{\omega_{\varepsilon, \delta_{0}}}=\varliminf_{\delta_{0} \rightarrow 0}^{\lim }\left\|\alpha_{\varepsilon}\right\|_{\varepsilon, \delta_{0}}^{2} .
$$

These inequalities lead to the desired estimate in the proposition.

For simplicity, we use the same notation $\left\{u_{\varepsilon, \delta}\right\}_{\delta>0}$ for the subsequence $\left\{u_{\varepsilon, \delta_{\nu}}\right\}_{\nu=1}^{\infty}$ in Proposition 5.4. We fix $\varepsilon_{0}>0$ and consider the weak limit of $\alpha_{\varepsilon}$ in the fixed $L^{2}$-space $L_{(2)}^{n, q}(F)_{h_{\varepsilon_{0}}, \omega}$. For a sufficiently small $\varepsilon>0$, we have

$$
\left\|\alpha_{\varepsilon}\right\|_{h_{\varepsilon_{0}}, \omega} \leq\left\|\alpha_{\varepsilon}\right\|_{h_{\varepsilon}, \omega} \leq\|u\|_{h, \omega}
$$

by property (b) and Proposition 5.4. By taking a subsequence of $\left\{\alpha_{\varepsilon}\right\}_{\varepsilon>0}$, we may assume that $\alpha_{\varepsilon}$ weakly converges to some $\alpha$ in $L_{(2)}^{n, q}(F)_{h_{\varepsilon_{0}}, \omega}$.

Proposition 5.6. If the weak limit $\alpha$ is zero in $L_{(2)}^{n, q}(F)_{h_{\varepsilon_{0}}, \omega}$, then the cohomology class $\eta$ is zero in $H^{q}\left(X, K_{X} \otimes F \otimes \mathcal{J}(h)\right)$.

Proof of Proposition 5.6. For every $\delta$ with $0<\delta \leq \delta_{0}$, we can easily check

$$
u-u_{\varepsilon, \delta} \in \operatorname{Im} \bar{\partial} \text { in } L_{(2)}^{n, q}(F)_{\varepsilon, \delta} \subset \operatorname{Im} \bar{\partial} \text { in } L_{(2)}^{n, q}(F)_{\varepsilon, \delta_{0}}
$$

from the construction of $u_{\varepsilon, \delta}$. As $\delta \rightarrow 0$, we obtain

$$
u-\alpha_{\varepsilon} \in \operatorname{Im} \bar{\partial} \operatorname{in} L_{(2)}^{n, q}(F)_{\varepsilon, \delta_{0}}
$$

by Lemma 2.6 and Proposition 5.4. We remark that $\operatorname{Im} \bar{\partial}$ is a closed subspace (see [MaS4, Proposition 5.8]). On the other hand, we have the following commutative diagram:

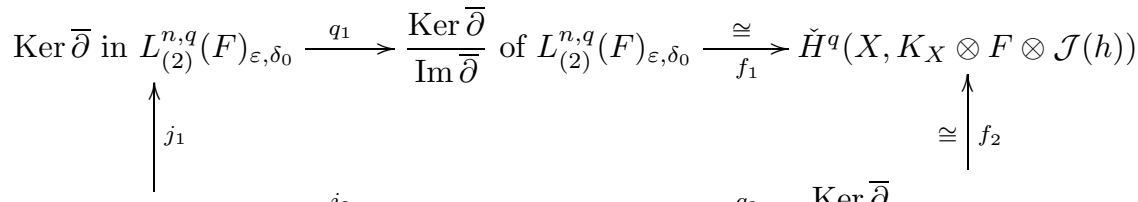

$\operatorname{Ker} \bar{\partial}$ in $L_{(2)}^{n, q}(F)_{h_{\varepsilon}, \omega} \stackrel{j_{2}}{\longrightarrow} \operatorname{Ker} \bar{\partial}$ in $L_{(2)}^{n, q}(F)_{h_{\varepsilon_{0}}, \omega} \stackrel{q_{2}}{\longrightarrow} \frac{\operatorname{Ker} \bar{\partial}}{\operatorname{Im} \bar{\partial}}$ of $L_{(2)}^{n, q}(F)_{h_{\varepsilon_{0}}, \omega}$.

Here $j_{1}, j_{2}$ are the natural inclusions, $q_{1}, q_{2}$ are the natural quotient maps, and $f_{1}, f_{2}$ are the de Rham-Weil isomorphisms (see MaS4, Proposition 5.5] for the construction). Strictly speaking, $f_{1}$ is an isomorphism to $\check{H}^{q}\left(X, K_{X} \otimes F \otimes \mathcal{J}\left(h_{\varepsilon}\right)\right)$, but which coincides with $\check{H}^{q}\left(X, K_{X} \otimes F \otimes \mathcal{J}(h)\right)$ by property (c). To check that $j_{2}$ is well-defined, we have to see that $\bar{\partial} w=0$ on $Y_{\varepsilon_{0}}$ if $\bar{\partial} w=0$ on $Y_{\varepsilon}$. By the 
$L^{2}$-integrability and [D4, (7.3) Lemma, Chapter VIII], the equality $\bar{\partial} w=0$ can be extended from $Y_{\varepsilon}$ to $X$ (in particular $Y_{\varepsilon_{0}}$ ). The key point here is the $L^{2}$-integrability with respect to $\omega\left(\right.$ not $\left.\omega_{\varepsilon, \delta}\right)$.

Since $j_{2}\left(u-\alpha_{\varepsilon}\right)$ weakly converges to $j_{2}(u-\alpha)$ and the $\bar{\partial}$-cohomology is finite dimensional, we obtain

$$
\lim _{\varepsilon \rightarrow 0} q_{2}\left(u-\alpha_{\varepsilon}\right)=q_{2}(u-\alpha)=q_{2}(u)
$$

by Lemma 2.5 and the assumption $\alpha=0$. On the other hand, it follows that $q_{1}\left(u-\alpha_{\varepsilon}\right)=0$ from the first half argument. Hence, we have $q_{2}(u)=0$, that is, $u \in \operatorname{Im} \bar{\partial} \subset L_{(2)}^{n, q}(F)_{h_{\varepsilon_{0}}, \omega}$. From $q_{2}(u)=0$, we can prove the conclusion, that is, $u \in \operatorname{Im} \bar{\partial} \subset L_{(2)}^{n, q}(F)_{h, \omega}$. Indeed, we can obtain $q_{3}(u)=0$ (which leads to the conclusion) by the following commutative diagram:

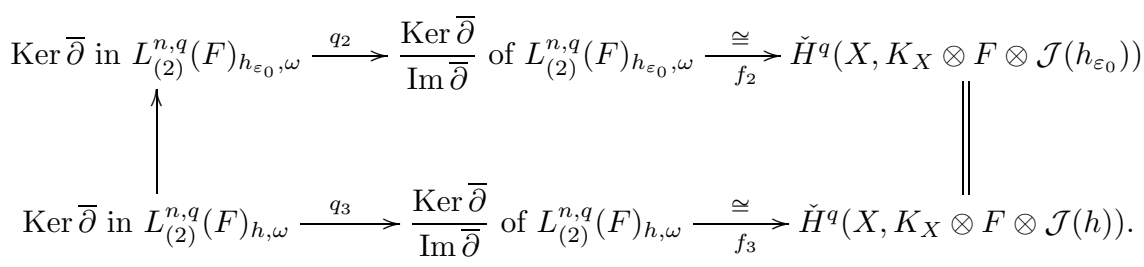

At the end of this step, we prove Proposition 5.7

Proposition 5.7. If we have

$$
\varliminf_{\varepsilon \rightarrow 0} \varliminf_{\delta \rightarrow 0}\left\|s u_{\varepsilon, \delta}\right\|_{h_{\varepsilon} h_{M}, \omega_{\varepsilon, \delta}}=0,
$$

then the weak limit $\alpha$ is zero. In particular, the cohomology class $\eta$ is zero by Proposition 5.6.

Proof of Proposition 5.7. In the proof, we compare the norm of $u_{\varepsilon, \delta}$ with the norm of $s u_{\varepsilon, \delta}$. For this purpose, we define $Y_{\varepsilon_{0}}^{k}$ to be

$$
Y_{\varepsilon_{0}}^{k}:=\left\{\left.y \in Y_{\varepsilon_{0}}|| s\right|_{h_{M}}>1 / k \text { at } y\right\}
$$

for $k \gg 0$. Note the subset $Y_{\varepsilon_{0}}^{k}$ is an open set in $Y_{\varepsilon_{0}}$. It follows that the restriction $\left.\alpha_{\varepsilon}\right|_{Y_{\varepsilon_{0}}^{k}}$ also weakly converges to $\left.\alpha\right|_{Y_{\varepsilon_{0}}^{k}}$ in $L_{(2)}^{n, q}\left(Y_{\varepsilon_{0}}^{k}, F\right)_{h_{\varepsilon_{0}}, \omega}$ since the restriction map $L_{(2)}^{n, q}(F)_{h_{\varepsilon_{0}}, \omega} \rightarrow L_{(2)}^{n, q}\left(Y_{\varepsilon_{0}}^{k}, F\right)_{h_{\varepsilon_{0}}, \omega}$ is a bounded operator and $\alpha_{\varepsilon}$ weakly converges to $\alpha$ in $L_{(2)}^{n, q}(F)_{h_{\varepsilon_{0}}, \omega}$. Since the norm is lower semicontinuous with respect to the weak convergence, we obtain the estimate for the $L^{2}$-norm on $Y_{\varepsilon_{0}}^{k}$

$$
\|\alpha\|_{Y_{\varepsilon_{0}}^{k}, h_{\varepsilon_{0}}, \omega} \leq \varliminf_{\varepsilon \rightarrow 0}\left\|\alpha_{\varepsilon}\right\|_{Y_{\varepsilon_{0}}^{k}, h_{\varepsilon_{0}}, \omega} \leq \varliminf_{\varepsilon \rightarrow 0}\left\|\alpha_{\varepsilon}\right\|_{Y_{\varepsilon_{0}}^{k}, h_{\varepsilon}, \omega}
$$

by property (b). By the same argument, the restriction $\left.u_{\varepsilon, \delta}\right|_{Y_{\varepsilon_{0}}^{k}}$ weakly converges to $\left.\alpha_{\varepsilon}\right|_{Y_{\varepsilon_{0}}^{k}}$ in $L_{(2)}^{n, q}\left(Y_{\varepsilon_{0}}^{k}, F\right)_{\varepsilon, \delta_{0}}$, and thus we obtain

$$
\left\|\alpha_{\varepsilon}\right\|_{Y_{\varepsilon_{0}}^{k}, \varepsilon, \delta_{0}} \leq \varliminf_{\delta \rightarrow 0}\left\|u_{\varepsilon, \delta}\right\|_{Y_{\varepsilon_{0}}^{k}, \varepsilon, \delta_{0}} \leq \varliminf_{\delta \rightarrow 0}\left\|u_{\varepsilon, \delta}\right\|_{Y_{\varepsilon_{0}}^{k}, \varepsilon, \delta}
$$

by Lemma 2.4. As $\delta_{0} \rightarrow 0$ in the above inequality, we have

$$
\left\|\alpha_{\varepsilon}\right\|_{Y_{\varepsilon_{0}}^{k}, h_{\varepsilon}, \omega} \leq \varliminf_{\delta_{0} \rightarrow 0}\left\|\alpha_{\varepsilon}\right\|_{Y_{\varepsilon_{0}}^{k}, \varepsilon, \delta_{0}} \leq \varliminf_{\delta \rightarrow 0}\left\|u_{\varepsilon, \delta}\right\|_{Y_{\varepsilon_{0}}^{k}, \varepsilon, \delta}
$$


by Fatou's lemma (see the argument in Proposition 5.4). These inequalities yield

$$
\|\alpha\|_{Y_{\varepsilon_{0}}^{k}, h_{\varepsilon_{0}}, \omega} \leq \varliminf_{\varepsilon \rightarrow 0} \frac{\lim _{\delta \rightarrow 0}}{\delta \rightarrow u_{\varepsilon, \delta}} \|_{Y_{\varepsilon_{0}}^{k}, \varepsilon, \delta} .
$$

On the other hand, it follows that

$$
\left\|u_{\varepsilon, \delta}\right\|_{Y_{\varepsilon_{0}}^{k}, \varepsilon, \delta} \leq k\left\|s u_{\varepsilon, \delta}\right\|_{Y_{\varepsilon_{0}}^{k}, h_{\varepsilon} h_{M}, \omega_{\varepsilon, \delta}} \leq k\left\|s u_{\varepsilon, \delta}\right\|_{h_{\varepsilon} h_{M}, \omega_{\varepsilon, \delta}}
$$

since the inequality $1 / k<|s|_{h_{M}}$ holds on $Y_{\varepsilon_{0}}^{k}$. This implies that $\alpha=0$ on $Y_{\varepsilon_{0}}^{k}$ for an arbitrary $k \gg 0$. From $\bigcup_{k \gg 0} Y_{\varepsilon_{0}}^{k}=Y_{\varepsilon_{0}} \backslash\{s=0\}$, we obtain the desired conclusion.

Step 3. The purpose of this step is to prove Proposition 5.8

\section{Proposition 5.8.}

$$
\lim _{\varepsilon \rightarrow 0} \varlimsup_{\delta \rightarrow 0}\left\|\bar{\partial}_{\varepsilon, \delta}^{*} s u_{\varepsilon, \delta}\right\|_{h_{\varepsilon} h_{M}, \omega_{\varepsilon, \delta}}=0 .
$$

Proof of Proposition 5.8. In the proof, we will often use (5.3). By applying BochnerKodaira-Nakano's identity and the density lemma to $u_{\varepsilon, \delta}$ and $s u_{\varepsilon, \delta}$ (see MaS1, Proposition 2.8]), we obtain

$$
\begin{aligned}
& 0=\left\langle\sqrt{-1} \Theta_{h_{\varepsilon}}(F) \Lambda_{\omega_{\varepsilon, \delta}} u_{\varepsilon, \delta}, u_{\varepsilon, \delta}\right\rangle_{\varepsilon, \delta}+\left\|D_{\varepsilon, \delta}^{\prime *} u_{\varepsilon, \delta}\right\|_{\varepsilon, \delta}^{2} \\
& \left\|\bar{\partial}_{\varepsilon, \delta}^{*} s u_{\varepsilon, \delta}\right\|_{h_{\varepsilon} h_{M}, \omega_{\varepsilon, \delta}}^{2} \\
& \quad=\left\langle\sqrt{-1} \Theta_{h_{\varepsilon} h_{M}}(F \otimes M) \Lambda_{\omega_{\varepsilon, \delta}} s u_{\varepsilon, \delta}, s u_{\varepsilon, \delta}\right\rangle_{h_{\varepsilon} h_{M}, \omega_{\varepsilon, \delta}}+\left\|D_{\varepsilon, \delta}^{\prime *} s u_{\varepsilon, \delta}\right\|_{h_{\varepsilon} h_{M}, \omega_{\varepsilon, \delta}}^{2},
\end{aligned}
$$

where $D_{\varepsilon, \delta}^{\prime *}$ is the adjoint operator of the $(1,0)$-part of the Chern connection $D_{h_{\varepsilon}}$. Here we used the fact that $u_{\varepsilon, \delta}$ is harmonic and $\bar{\partial}\left(s u_{\varepsilon, \delta}\right)=s \bar{\partial} u_{\varepsilon, \delta}=0$. Now we have

$$
\sqrt{-1} \Theta_{h_{\varepsilon}}(F) \geq b \sqrt{-1} \Theta_{h_{M}}(M)-\varepsilon \omega \geq-\varepsilon \omega \geq-\varepsilon \omega_{\varepsilon, \delta}
$$

by property (d) and property (B). Hence, the integrand $g_{\varepsilon, \delta}$ of the first term of (5.4) satisfies

$$
-\varepsilon q\left|u_{\varepsilon, \delta}\right|_{\varepsilon, \delta}^{2} \leq g_{\varepsilon, \delta}:=\left\langle\sqrt{-1} \Theta_{h_{\varepsilon}}(F) \Lambda_{\omega_{\varepsilon, \delta}} u_{\varepsilon, \delta}, u_{\varepsilon, \delta}\right\rangle_{\varepsilon, \delta}
$$

For the precise argument, see MaS4, Step 2 in the proof of Theorem 3.1]. Then by (5.4), we can easily see

$$
\begin{aligned}
\lim _{\varepsilon \rightarrow 0} & \varlimsup_{\delta \rightarrow 0}\left(\int_{\left\{g_{\varepsilon, \delta} \geq 0\right\}} g_{\varepsilon, \delta} d V_{\omega_{\varepsilon, \delta}}+\left\|D_{\varepsilon, \delta}^{\prime *} u_{\varepsilon, \delta}\right\|_{\varepsilon, \delta}^{2}\right) \\
& =\lim _{\varepsilon \rightarrow 0} \varlimsup_{\delta \rightarrow 0}\left(-\int_{\left\{g_{\varepsilon, \delta} \leq 0\right\}} g_{\varepsilon, \delta} d V_{\omega_{\varepsilon, \delta}}\right) \\
& \leq \lim _{\varepsilon \rightarrow 0} \varlimsup_{\delta \rightarrow 0}\left(\varepsilon q \int_{\left\{g_{\varepsilon, \delta} \leq 0\right\}}\left|u_{\varepsilon, \delta}\right|_{\varepsilon, \delta}^{2} d V_{\omega_{\varepsilon, \delta}}\right) \\
& \leq \lim _{\varepsilon \rightarrow 0} \varlimsup_{\delta \rightarrow 0}\left(\varepsilon q\left\|u_{\varepsilon, \delta}\right\|_{\varepsilon, \delta}^{2}\right)=0 .
\end{aligned}
$$

Here we used (5.3) in the last equality. 
On the other hand, by $\sqrt{-1} \Theta_{h_{\varepsilon}}(F) \geq b \sqrt{-1} \Theta_{h_{M}}(M)-\varepsilon \omega_{\varepsilon, \delta}$, we have

$$
\begin{aligned}
& \left\langle\sqrt{-1} \Theta_{h_{\varepsilon} h_{M}}(F \otimes M) \Lambda_{\omega_{\varepsilon, \delta}} s u_{\varepsilon, \delta}, s u_{\varepsilon, \delta}\right\rangle_{h_{\varepsilon} h_{M}, \omega_{\varepsilon, \delta}} \\
\leq & \left(1+\frac{1}{b}\right) \int_{Y_{\varepsilon}}|s|_{h_{M}}^{2} g_{\varepsilon, \delta} d V_{\omega_{\varepsilon, \delta}}+\frac{\varepsilon q}{b} \int_{Y_{\varepsilon}}|s|_{h_{M}}^{2}\left|u_{\varepsilon, \delta}\right|_{\varepsilon, \delta}^{2} d V_{\omega_{\varepsilon, \delta}} \\
\leq & \left(1+\frac{1}{b}\right) \sup _{X}|s|_{h_{M}}^{2}\left\{\int_{\left\{g_{\varepsilon, \delta} \geq 0\right\}} g_{\varepsilon, \delta} d V_{\omega_{\varepsilon, \delta}}+\frac{\varepsilon q}{b} \sup _{X}|s|_{h_{M}}^{2}\left\|u_{\varepsilon, \delta}\right\|_{\varepsilon, \delta}^{2}\right\} .
\end{aligned}
$$

Furthermore, since $D_{\varepsilon, \delta}^{\prime *}$ can be expressed as $D_{\varepsilon, \delta}^{\prime *}=-* \bar{\partial} *$ by the Hodge star operator $*$ with respect to $\omega_{\varepsilon, \delta}$, we have

$$
\left\|D_{\varepsilon, \delta}^{\prime *} s u_{\varepsilon, \delta}\right\|_{h_{\varepsilon} h_{M}, \omega_{\varepsilon, \delta}}^{2}=\left\|s D_{\varepsilon, \delta}^{\prime *} u_{\varepsilon, \delta}\right\|_{h_{\varepsilon} h_{M}, \omega_{\varepsilon, \delta}}^{2} \leq \sup _{X}|s|_{h_{M}}^{2}\left\|D_{\varepsilon, \delta}^{\prime *} u_{\varepsilon, \delta}\right\|_{\varepsilon, \delta}^{2}
$$

The right-hand side of (5.5) can be shown to converge to zero by the first half argument and these inequalities.

Step 4. In this step, we construct solutions $v_{\varepsilon, \delta}$ of the $\bar{\partial}$-equation $\bar{\partial} v_{\varepsilon, \delta}=s u_{\varepsilon, \delta}$ with suitable $L^{2}$-norm, and we finish the proof of Theorem 5.1. The proof of the following proposition is a slight variant of that of [MaS4, Theorem 5.9].

Proposition 5.9. There exist $F$-valued $(n, q-1)$-forms $w_{\varepsilon, \delta}$ on $Y_{\varepsilon}$ with the following properties:

- $\bar{\partial} w_{\varepsilon, \delta}=u-u_{\varepsilon, \delta}$.

- $\overline{\lim }_{\delta \rightarrow 0}\left\|w_{\varepsilon, \delta}\right\|_{\varepsilon, \delta}$ can be bounded by a constant independent of $\varepsilon$.

Before we begin to prove Proposition 5.9, we recall the content in MaS4, Section $5]$ with our notation. For a finite open cover $\mathcal{U}:=\left\{B_{i}\right\}_{i \in I}$ of $X$ by sufficiently small Stein open sets $B_{i}$, we can construct

$$
f_{\varepsilon, \delta}: \operatorname{Ker} \bar{\partial} \text { in } L_{(2)}^{n, q}(F)_{\varepsilon, \delta} \longrightarrow \operatorname{Ker} \mu \text { in } C^{q}\left(\mathcal{U}, K_{X} \otimes F \otimes \mathcal{J}\left(h_{\varepsilon}\right)\right)
$$

such that $f_{\varepsilon, \delta}$ induces the de Rham-Weil isomorphism

$$
\overline{f_{\varepsilon, \delta}}: \frac{\operatorname{Ker} \bar{\partial}}{\operatorname{Im} \bar{\partial}} \text { of } L_{(2)}^{n, q}(F)_{\varepsilon, \delta} \cong \frac{\operatorname{Ker} \mu}{\operatorname{Im} \mu} \text { of } C^{q}\left(\mathcal{U}, K_{X} \otimes F \otimes \mathcal{J}\left(h_{\varepsilon}\right)\right) .
$$

Here $C^{q}\left(\mathcal{U}, K_{X} \otimes F \otimes \mathcal{J}\left(h_{\varepsilon}\right)\right)$ is the space of $q$-cochains calculated by $\mathcal{U}$ and $\mu$ is the coboundary operator. We remark that $C^{q}\left(\mathcal{U}, K_{X} \otimes F \otimes \mathcal{J}\left(h_{\varepsilon}\right)\right)$ is a Fréchet space with respect to the seminorm $p_{K_{i_{0} \ldots i_{q}}}(\bullet)$ defined to be

$$
p_{K_{i_{0} \ldots i_{q}}}\left(\left\{\beta_{i_{0} \ldots i_{q}}\right\}\right)^{2}:=\int_{K_{i_{0} \ldots i_{q}}}\left|\beta_{i_{0} \ldots i_{q}}\right|_{h_{\varepsilon}, \omega}^{2} d V_{\omega}
$$

for a relatively compact set $K_{i_{0} \ldots i_{q}} \Subset B_{i_{0} \ldots i_{q}}:=B_{i_{0}} \cap \cdots \cap B_{i_{q}}$ (see [MaS4, Theorem $5.3])$. The construction of $f_{\varepsilon, \delta}$ is essentially the same as in the proof of MaS4, Proposition 5.5]. The only difference is that we use Lemma 5.12 instead of [MaS4, Lemma 5.4] when we locally solve the $\bar{\partial}$-equation to construct $f_{\varepsilon, \delta}$. Lemma 5.12 will be given at the end of this step. We prove Proposition 5.9 by replacing some constants appearing in the proof of [MaS4, Theorem 5.9] with $C_{\varepsilon, \delta}$ appearing in Lemma 5.12 
Proof of Proposition [5.9. We put $U_{\varepsilon, \delta}:=u-u_{\varepsilon, \delta} \in \operatorname{Im} \bar{\partial} \subset L_{(2)}^{n, q}(F)_{\varepsilon, \delta}$. Then there exist the $F$-valued $(n, q-k-1)$-forms $\beta_{i_{0} \ldots i_{k}}^{\varepsilon, \delta}$ on $B_{i_{0} \ldots i_{k}} \backslash Z_{\varepsilon}$ satisfying

$$
(*) \begin{cases}\bar{\partial} \beta_{i_{0}}^{\varepsilon, \delta} & =\left.U_{\varepsilon, \delta}\right|_{B_{i_{0}} \backslash Z_{\varepsilon}}, \\ \bar{\partial}\left\{\beta_{i_{0} i_{1}}^{\varepsilon, \delta}\right\} & =\mu\left\{\beta_{i_{0}}^{\varepsilon, \delta}\right\}, \\ \bar{\partial}\left\{\beta_{i_{0} i_{1} i_{2}}^{\varepsilon, \delta}\right\} & =\mu\left\{\beta_{i_{0} i_{1}}^{\varepsilon, \delta}\right\}, \\ \bar{\partial}\left\{\beta_{i_{0} \ldots i_{q-1}}^{\varepsilon, \delta}\right\} & =\mu\left\{\beta_{i_{0} \ldots i_{q-2}}^{\varepsilon, \delta}\right\}, \\ f_{\varepsilon, \delta}\left(U_{\varepsilon, \delta}\right) & =\mu\left\{\beta_{i_{0} \ldots i_{q-1}}^{\varepsilon, \delta}\right\} .\end{cases}
$$

Here $\beta_{i_{0} \ldots i_{k}}^{\varepsilon, \delta}$ is the solution of the above equation whose norm is minimum among all the solutions (see the construction of $f_{\varepsilon, \delta}$ in [MaS4, Proposition 5.5]). For example, $\beta_{i_{0}}^{\varepsilon, \delta}$ is the solution of $\bar{\partial} \beta_{i_{0}}^{\varepsilon, \delta}=U_{\varepsilon, \delta}$ on $B_{i_{0}} \backslash Z_{\varepsilon}$ whose norm $\left\|\beta_{i_{0}}^{\varepsilon, \delta}\right\|_{\varepsilon, \delta}$ is minimum among all the solutions. In particular $\left\|\beta_{i_{0}}^{\varepsilon, \delta}\right\|_{\varepsilon, \delta}^{2} \leq C_{\varepsilon, \delta}\left\|U_{\varepsilon, \delta}\right\|_{B_{i_{0}}, \varepsilon, \delta}^{2} \leq C_{\varepsilon, \delta}\left\|U_{\varepsilon, \delta}\right\|_{\varepsilon, \delta}^{2}$ holds for some constant $C_{\varepsilon, \delta}$ by Lemma [5.12. where $C_{\varepsilon, \delta}$ is a constant such that $\varlimsup_{\delta \rightarrow 0} C_{\varepsilon, \delta}$ (is finite and) is independent of $\varepsilon$. Similarly, $\beta_{i_{0} i_{1}}^{\varepsilon, \delta}$ is the solution of $\bar{\partial} \beta_{i_{0} i_{1}}^{\varepsilon, \delta}=\left(\beta_{i_{1}}^{\varepsilon, \delta}-\beta_{i_{0}}^{\varepsilon, \delta}\right)$ on $B_{i_{0} i_{1}} \backslash Z_{\varepsilon}$ and the norm

$$
\left\|\beta_{i_{0} i_{1}}^{\varepsilon, \delta}\right\|_{\varepsilon, \delta}^{2}:=\int_{B_{i_{0} i_{1}} \backslash Z_{\varepsilon}}\left|\beta_{i_{0} i_{1}}^{\varepsilon, \delta}\right|_{\varepsilon, \delta}^{2} d V_{\varepsilon, \delta}
$$

is minimum among all the solutions. In particular, $\left\|\beta_{i_{0} i_{1}}^{\varepsilon, \delta}\right\|_{\varepsilon, \delta}^{2} \leq D_{\varepsilon, \delta}\left\|\left(\beta_{i_{1}}^{\varepsilon, \delta}-\beta_{i_{0}}^{\varepsilon, \delta}\right)\right\|_{\varepsilon, \delta}^{2}$ holds for some constant $D_{\varepsilon, \delta}$ by Lemma 5.12. Of course $D_{\varepsilon, \delta}$ is a constant such that $\varlimsup_{\delta \rightarrow 0} D_{\varepsilon, \delta}$ (is finite and) is independent of $\varepsilon$. Hence we have

$$
\left\|\beta_{i_{0} i_{1}}^{\varepsilon, \delta}\right\|_{\varepsilon, \delta} \leq D_{\varepsilon, \delta}^{1 / 2}\left\|\left(\beta_{i_{1}}^{\varepsilon, \delta}-\beta_{i_{0}}^{\varepsilon, \delta}\right)\right\|_{\varepsilon, \delta} \leq 2 C_{\varepsilon, \delta}^{1 / 2} D_{\varepsilon, \delta}^{1 / 2}\left\|U_{\varepsilon, \delta}\right\|_{\varepsilon, \delta} \leq 4 C_{\varepsilon, \delta}^{1 / 2} D_{\varepsilon, \delta}^{1 / 2}\|u\|_{h, \omega}
$$

by (5.3). From now on, the notation $C_{\varepsilon, \delta}$ denotes a (possibly different) constant such that $\varlimsup_{\delta \rightarrow 0} C_{\varepsilon, \delta}$ can be bounded by a constant independent of $\varepsilon$. By repeating this process, we have

$$
\left\|\beta_{i_{0} \ldots i_{k}}^{\varepsilon, \delta}\right\|_{\varepsilon, \delta}^{2} \leq C_{\varepsilon, \delta}\|u\|_{h, \omega}^{2} .
$$

Moreover, by property (c), we have

$\alpha_{\varepsilon, \delta}:=f_{\varepsilon, \delta}\left(U_{\varepsilon, \delta}\right)=\mu\left\{\beta_{i_{0} \ldots i_{q-1}}^{\varepsilon, \delta}\right\} \in C^{q}\left(\mathcal{U}, K_{X} \otimes F \otimes \mathcal{J}\left(h_{\varepsilon}\right)\right)=C^{q}\left(\mathcal{U}, K_{X} \otimes F \otimes \mathcal{J}(h)\right)$.

Claim. There exist subsequences $\left\{\varepsilon_{k}\right\}_{k=1}^{\infty}$ and $\left\{\delta_{\ell}\right\}_{\ell=1}^{\infty}$ with the following properties:

- $\alpha_{\varepsilon_{k}, \delta_{\ell}} \rightarrow \alpha_{\varepsilon_{k}, 0}$ in $C^{q}\left(\mathcal{U}, K_{X} \otimes F \otimes \mathcal{J}(h)\right)$ as $\delta_{\ell} \rightarrow 0$.

- $\alpha_{\varepsilon_{k}, 0} \rightarrow \alpha_{0,0}$ in $C^{q}\left(\mathcal{U}, K_{X} \otimes F \otimes \mathcal{J}(h)\right)$ as $\varepsilon_{k} \rightarrow 0$.

Moreover, the limit $\alpha_{0,0}$ belongs to $B^{q}\left(\mathcal{U}, K_{X} \otimes F \otimes \mathcal{J}(h)\right):=\operatorname{Im} \mu$.

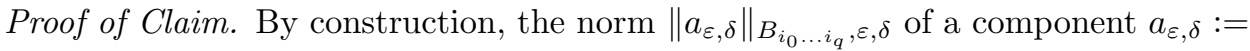
$\alpha_{i_{0} \ldots i_{q}}^{\varepsilon, \delta}$ of $\alpha_{\varepsilon, \delta}=\left\{\alpha_{i_{0} \ldots i_{q}}^{\varepsilon, \delta}\right\}$ can be bounded by a constant $C_{\varepsilon, \delta}$. Note that $a_{\varepsilon, \delta}$ can be regarded as a holomorphic function on $B_{i_{0} \ldots i_{q}} \backslash Z_{\varepsilon}$ with bounded $L^{2}$-norm since

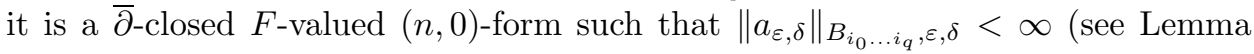
2.4). Hence $a_{\varepsilon, \delta}$ can be extended from $B_{i_{0} \ldots i_{q}} \backslash Z_{\varepsilon}$ to $B_{i_{0} \ldots i_{q}}$ by the Riemann extension theorem. The sup-norm $\sup _{K}\left|a_{\varepsilon, \delta}\right|$ is uniformly bounded with respect to $\delta$ for every $K \Subset B_{i_{0} \ldots i_{q}}$ since the local sup-norm of holomorphic functions can be bounded by the $L^{2}$-norm. By Montel's theorem, we can take a subsequence 
$\left\{\delta_{\ell}\right\}_{\ell=1}^{\infty}$ with the first property. This subsequence may depend on $\varepsilon$, but we can take $\left\{\delta_{\ell}\right\}_{\ell=1}^{\infty}$ independent of (countably many) $\varepsilon$. Then the norm of the limit $a_{\varepsilon, 0}$ is uniformly bounded with respect to $\varepsilon$ since $\varlimsup_{\delta \rightarrow 0} C_{\varepsilon, \delta}$ can be bounded by a constant independent of $\varepsilon$ (see Lemma 5.12). Therefore, by applying Montel's theorem again, we can take a subsequence $\left\{\varepsilon_{k}\right\}_{k=1}^{\infty}$ with the second property. We remark that the convergence with respect to the sup-norm implies the convergence with respect to the local $L^{2}$-norm $p_{K}(\bullet)$ (see [MaS4, Lemma 5.2]).

It is easy to check the latter conclusion. Indeed, it follows that $\alpha_{\varepsilon, \delta}=f_{\varepsilon, \delta}\left(U_{\varepsilon, \delta}\right) \in$ $\operatorname{Im} \mu$ since $U_{\varepsilon, \delta} \in \operatorname{Im} \bar{\partial} \subset L_{(2)}^{n, q}(F)_{\varepsilon, \delta}$ and $f_{\varepsilon, \delta}$ induces the de Rham-Weil isomorphism. By MaS4, Lemma 5.7], the subspace $\operatorname{Im} \mu$ is closed. Therefore, we obtain the latter conclusion.

Now, we construct solutions $\gamma_{\varepsilon, \delta}$ of the equation $\mu \gamma_{\varepsilon, \delta}=\alpha_{\varepsilon, \delta}$ with suitable $L^{2}$ norm. For simplicity, we continue to use the same notation for the subsequences in Claim. By the latter conclusion of the claim, there exists $\gamma \in C^{q-1}\left(\mathcal{U}, K_{X} \otimes F \otimes\right.$ $\mathcal{J}(h))$ such that $\mu \gamma=\alpha_{0,0}$. The coboundary operator

$$
\mu: C^{q-1}\left(\mathcal{U}, K_{X} \otimes F \otimes \mathcal{J}(h)\right) \rightarrow B^{q}\left(\mathcal{U}, K_{X} \otimes F \otimes \mathcal{J}(h)\right)=\operatorname{Im} \mu
$$

is a surjective bounded operator between Fréchet spaces (see [MaS4, Lemma 5.7]), and thus it is an open map by the open mapping theorem. Therefore $\mu\left(\Delta_{K}\right)$ is an open neighborhood of the limit $\alpha_{0,0}$ in $\operatorname{Im} \mu$, where $\Delta_{K}$ is the open bounded neighborhood of $\gamma$ in $C^{q-1}\left(\mathcal{U}, K_{X} \otimes F \otimes \mathcal{J}(h)\right)$ defined to be

$$
\Delta_{K}:=\left\{\beta \in C^{q-1}\left(\mathcal{U}, K_{X} \otimes F \otimes \mathcal{J}(h)\right) \mid p_{K_{i_{0} \ldots i_{q-1}}}(\beta-\gamma)<1\right\}
$$

for a family $K:=\left\{K_{i_{0} \ldots i_{q-1}}\right\}$ of relatively compact sets $K_{i_{0} \ldots i_{q-1}} \Subset B_{i_{0} \ldots i_{q-1}}$. We have $\alpha_{\varepsilon, \delta} \in \mu\left(\Delta_{K}\right)$ for sufficiently small $\varepsilon, \delta>0$ since $\alpha_{\varepsilon, \delta}$ converges to $\alpha_{0,0}$. Since $\Delta_{K}$ is bounded, we can obtain $\gamma_{\varepsilon, \delta} \in C^{q-1}\left(\mathcal{U}, K_{X} \otimes F \otimes \mathcal{J}(h)\right)$ such that

$$
\mu \gamma_{\varepsilon, \delta}=\alpha_{\varepsilon, \delta} \quad \text { and } \quad p_{K_{i_{0} \ldots i_{q-1}}}\left(\gamma_{\varepsilon, \delta}\right)^{2} \leq C_{K}
$$

for some positive constant $C_{K}$. The above constant $C_{K}$ depends on the choice of $K, \gamma$, but does not depend on $\varepsilon, \delta$.

By the same argument as in MaS4, Claim 5.11 and Claim 5.13], we can obtain $F$-valued $(n, q-1)$-forms $w_{\varepsilon, \delta}$ with the desired properties. The strategy is as follows: The inverse map $\overline{g_{\varepsilon, \delta}}$ of $\overline{f_{\varepsilon, \delta}}$ is explicitly constructed by using a partition of unity (see the proof of [MaS4, Proposition 5.5] and [MaS4, Remark 5.6]). We can easily see that $g_{\varepsilon, \delta}\left(\mu \gamma_{\varepsilon, \delta}\right)=\bar{\partial} v_{\varepsilon, \delta}$ and $g_{\varepsilon, \delta}\left(\alpha_{\varepsilon, \delta}\right)=U_{\varepsilon, \delta}+\bar{\partial} \widetilde{v}_{\varepsilon, \delta}$ hold for some $v_{\varepsilon, \delta}$ and $\widetilde{v}_{\varepsilon, \delta}$ by the de Rham-Weil isomorphism. In particular, we have $U_{\varepsilon, \delta}=\bar{\partial}\left(v_{\varepsilon, \delta}-\widetilde{v}_{\varepsilon, \delta}\right)$ by $\mu \gamma_{\varepsilon, \delta}=\alpha_{\varepsilon, \delta}$. The important point here is that we can explicitly compute $v_{\varepsilon, \delta}$ and $\widetilde{v}_{\varepsilon, \delta}$ by using the partition of unity, $\beta_{i_{0} \ldots i_{k}}^{\varepsilon, \delta}$, and $\gamma_{\varepsilon, \delta}$. From this explicit expression, we obtain the $L^{2}$-estimate for $v_{\varepsilon, \delta}$ and $\widetilde{v}_{\varepsilon, \delta}$. See [MaS4, Claim 5.11 and 5.13] for the precise argument.

Proposition 5.10. There exist $F \otimes M$-valued $(n, q-1)$-forms $v_{\varepsilon, \delta}$ on $Y_{\varepsilon}$ with the following properties:

- $\bar{\partial} v_{\varepsilon, \delta}=s u_{\varepsilon, \delta}$.

- $\varlimsup_{\delta \rightarrow 0}\left\|v_{\varepsilon, \delta}\right\|_{h_{\varepsilon} h_{M}, \omega_{\varepsilon, \delta}}$ can be bounded by a constant independent of $\varepsilon$.

Proof of Proposition 5.10. Since the cohomology class of su is assumed to be zero in $H^{q}\left(X, K_{X} \otimes F \otimes \mathcal{J}(h) \otimes M\right)$, there exists an $F \otimes M$-valued $(n, q-1)$-form $v$ 
such that $\bar{\partial} v=s u$ and $\|v\|_{h, \omega}<\infty$. For $w_{\varepsilon, \delta}$ satisfying the properties in Proposition 5.9, by putting $v_{\varepsilon, \delta}:=-s w_{\varepsilon, \delta}+v$, we have $\bar{\partial} v_{\varepsilon, \delta}=s u_{\varepsilon, \delta}$. Furthermore, an easy computation yields

$$
\begin{aligned}
\left\|v_{\varepsilon, \delta}\right\|_{h_{\varepsilon} h_{M}, \omega_{\varepsilon, \delta}} & \leq\left\|s w_{\varepsilon, \delta}\right\|_{h_{\varepsilon} h_{M}, \omega_{\varepsilon, \delta}}+\|v\|_{h_{\varepsilon} h_{M}, \omega_{\varepsilon, \delta}} \\
& \leq \sup _{X}|s|_{h_{M}}\left\|w_{\varepsilon, \delta}\right\|_{\varepsilon, \delta}+\|v\|_{h_{\varepsilon} h_{M}, \omega_{\varepsilon, \delta}} .
\end{aligned}
$$

By Lemma 2.4, property (b), and property (B), we have $\|v\|_{h_{\varepsilon} h_{M}, \omega_{\varepsilon, \delta}} \leq\|v\|_{h, \omega}<\infty$. This completes the proof. 5.7).

The following proposition completes the proof of Theorem 5.1 (see Proposition

\section{Proposition 5.11.}

$$
\lim _{\varepsilon \rightarrow 0} \varlimsup_{\delta \rightarrow 0}\left\|s u_{\varepsilon, \delta}\right\|_{h_{\varepsilon} h_{M}, \omega_{\varepsilon, \delta}}=0 .
$$

Proof of Proposition 5.11. For the solution $v_{\varepsilon, \delta}$ satisfying the properties in Proposition 5.10, it is easy to see

$$
\begin{aligned}
\lim _{\varepsilon \rightarrow 0} \varlimsup_{\delta \rightarrow 0}\left\|s u_{\varepsilon, \delta}\right\|_{h_{\varepsilon} h_{M}, \omega_{\varepsilon, \delta}}^{2} & =\lim _{\varepsilon \rightarrow 0} \varlimsup_{\delta \rightarrow 0}\left\langle\bar{\partial}_{\varepsilon, \delta}^{*} s u_{\varepsilon, \delta}, v_{\varepsilon, \delta}\right\rangle_{h_{\varepsilon} h_{M}, \omega_{\varepsilon, \delta}} \\
& \leq \lim _{\varepsilon \rightarrow 0} \varlimsup_{\delta \rightarrow 0}\left\|\bar{\partial}_{\varepsilon, \delta}^{*} s u_{\varepsilon, \delta}\right\|_{h_{\varepsilon} h_{M}, \omega_{\varepsilon, \delta}}\left\|v_{\varepsilon, \delta}\right\|_{h_{\varepsilon} h_{M}, \omega_{\varepsilon, \delta} .}
\end{aligned}
$$

Proposition 5.8 and Proposition 5.10 assert that the right-hand side is zero.

We close this step with the following lemma:

Lemma 5.12 (cf. [D1, 4.1 Théorème]). Assume that $B$ is a Stein open set in $X$ such that $\omega_{\varepsilon, \delta}=\sqrt{-1} \partial \bar{\partial}\left(\Psi+\delta \Psi_{\varepsilon}\right)$ on a neighborhood of $\bar{B}$. Then for an arbitrary $\alpha \in \operatorname{Ker} \bar{\partial} \subset L_{(2)}^{n, q}\left(B \backslash Z_{\varepsilon}, F\right)_{\varepsilon, \delta}$, there exist $\beta \in L_{(2)}^{n, q-1}\left(B \backslash Z_{\varepsilon}, F\right)_{\varepsilon, \delta}$ and a positive constant $C_{\varepsilon, \delta}$ (independent of $\alpha$ ) such that

$$
\begin{aligned}
& \text { - } \bar{\partial} \beta=\alpha \quad \text { and } \quad\|\beta\|_{\varepsilon, \delta}^{2} \leq C_{\varepsilon, \delta}\|\alpha\|_{\varepsilon, \delta}^{2}, \\
& \text { - } \varlimsup_{\delta \rightarrow 0} C_{\varepsilon, \delta} \text { (is finite and) is independent of } \varepsilon .
\end{aligned}
$$

Proof of Lemma 5.12. We may assume $\varepsilon<1 / 2$ since $0<\varepsilon \ll 1$. For the singular Hermitian metric $H_{\varepsilon, \delta}$ on $F$ defined by $H_{\varepsilon, \delta}:=h_{\varepsilon} e^{-\left(\Psi+\delta \Psi_{\varepsilon}\right)}$, the curvature satisfies

$$
\begin{aligned}
\sqrt{-1} \Theta_{H_{\varepsilon, \delta}}(F) & =\sqrt{-1} \Theta_{h_{\varepsilon}}(F)+\sqrt{-1} \partial \bar{\partial}\left(\Psi+\delta \Psi_{\varepsilon}\right) \\
& \geq-\varepsilon \omega+\omega_{\varepsilon, \delta} \geq(1-\varepsilon) \omega_{\varepsilon, \delta} \geq \frac{1}{2} \omega_{\varepsilon, \delta}
\end{aligned}
$$

by property $(\mathrm{B})$ and $\sqrt{-1} \Theta_{h_{\varepsilon}}(F) \geq-\varepsilon \omega$. The $L^{2}$-norm $\|\alpha\|_{H_{\varepsilon, \delta}, \omega_{\varepsilon, \delta}}$ with respect to $H_{\varepsilon, \delta}$ and $\omega_{\varepsilon, \delta}$ is finite since the function $\Psi+\delta \Psi_{\varepsilon}$ is bounded and $\|\alpha\|_{\varepsilon, \delta}$ is finite. Therefore, from the standard $L^{2}$-method for the $\bar{\partial}$-equation (for example see [D1, 4.1 Théorème]), we obtain a solution $\beta$ of the $\bar{\partial}$-equation $\bar{\partial} \beta=\alpha$ with

$$
\|\beta\|_{H_{\varepsilon, \delta}, \omega_{\varepsilon, \delta}}^{2} \leq \frac{2}{q}\|\alpha\|_{H_{\varepsilon, \delta}, \omega_{\varepsilon, \delta}}^{2} .
$$

Then we can easily see that

$$
\|\beta\|_{\varepsilon, \delta}^{2} \leq \frac{2}{q} \frac{\sup _{B} e^{-\left(\Psi+\delta \Psi_{\varepsilon}\right)}}{\inf _{B} e^{-\left(\Psi+\delta \Psi_{\varepsilon}\right)}}\|\alpha\|_{\varepsilon, \delta}^{2} .
$$

This completes the proof by property (B). 
Remark 5.13. In Lemma 5.12, we take a solution $\beta_{0} \in L_{(2)}^{n, q-1}\left(B \backslash Z_{\varepsilon}, F\right)_{\varepsilon, \delta}$ of the equation $\bar{\partial} \beta=\alpha$. Then $\beta_{0}$ is uniquely decomposed as follows:

$$
\beta_{0}=\beta_{1}+\beta_{2} \quad \text { for } \beta_{1} \in \operatorname{Ker} \bar{\partial} \text { and } \beta_{2} \in(\operatorname{Ker} \bar{\partial})^{\perp} .
$$

We can easily check that $\beta_{2}$ is a unique solution of $\bar{\partial} \beta=\alpha$ whose norm is the minimum among all the solutions.

Thus we finish the proof of Theorem 5.1 .

\section{Twists By NAKano SEmipositive VeCtor BUndLES}

We have already known that some results for $K_{X}$ can be generalized for $K_{X} \otimes E$, where $E$ is a Nakano semipositive vector bundle on $X$ (see, for example, Ta, Mo, and $[\mathrm{Fs}]$ ). Let us recall the definition of Nakano semipositive vector bundles.

Definition 6.1 (Nakano semipositive vector bundles). Let $E$ be a holomorphic vector bundle on a complex manifold $X$. If $E$ admits a smooth Hermitian metric $h_{E}$ such that the curvature form $\sqrt{-1} \Theta_{h_{E}}(E)$ defines a positive semi-definite Hermitian form on each fiber of the vector bundle $E \otimes T_{X}$, where $T_{X}$ is the holomorphic tangent bundle of $X$, then $E$ is called a Nakano semipositive vector bundle.

Example 6.2 (Unitary flat vector bundles). Let $E$ be a holomorphic vector bundle on a complex manifold $X$. If $E$ admits a smooth Hermitian metric $h_{E}$ such that $\left(E, h_{E}\right)$ is flat, that is, $\sqrt{-1} \Theta_{h_{E}}(E)=0$, then $E$ is Nakano semipositive.

For the proof of Theorem 1.12, we need the following lemmas on Nakano semipositive vector bundles. However, these lemmas easily follow from the definition of Nakano semipositive vector bundles, and thus, we omit the proof.

Lemma 6.3. Let $E$ be a Nakano semipositive vector bundle on a complex manifold $X$. Let $H$ be a smooth divisor on $X$. Then $\left.E\right|_{H}$ is a Nakano semipositive vector bundle on $H$.

Lemma 6.4. Let $q: Z \rightarrow X$ be an étale morphism between complex manifolds. Let $\left(E, h_{E}\right)$ be a Nakano semipositive vector bundle on $X$. Then $\left(q^{*} E, q^{*} h_{E}\right)$ is a Nakano semipositive vector bundle on $Z$.

Proposition 6.5. Proposition 1.9 holds even when $K_{X}$ is replaced with $K_{X} \otimes E$, where $E$ is a Nakano semipositive vector bundle on $X$.

Proof. By Lemma 6.3 and Lemma 6.4, the proof of Proposition 1.9 in Section 4 works for $K_{X} \otimes E$.

Therefore, by Proposition 6.5 and the proof of Theorem 1.4 and Corollary 1.7 in Section 4, it is sufficient to prove the following theorem for Theorem 1.12 ,

Theorem 6.6 (Theorem A twisted by Nakano semipositive vector bundles). Let $E$ be a Nakano semipositive vector bundle on a compact Kähler manifold $X$. Let $F($ resp. $M)$ be a line bundle on a compact Kähler manifold $X$ with a singular Hermitian metric $h$ (resp. a smooth Hermitian metric $h_{M}$ ) satisfying

$$
\sqrt{-1} \Theta_{h_{M}}(M) \geq 0 \text { and } \sqrt{-1} \Theta_{h}(F)-b \sqrt{-1} \Theta_{h_{M}}(M) \geq 0 \text { for some } b>0 \text {. }
$$

Then for a (nonzero) section $s \in H^{0}(X, M)$, the multiplication map induced by $\otimes s$

$$
\times s: H^{q}\left(X, K_{X} \otimes E \otimes F \otimes \mathcal{J}(h)\right) \stackrel{\otimes s}{\longrightarrow} H^{q}\left(X, K_{X} \otimes E \otimes F \otimes \mathcal{J}(h) \otimes M\right)
$$


is injective for every $q$. Here $K_{X}$ is the canonical bundle of $X$ and $\mathcal{J}(h)$ is the multiplier ideal sheaf of $h$.

We will explain how to modify the proof of Theorem 5.1 for Theorem 6.6.

Proof. We replace $\left(F, h_{\varepsilon}\right)$ with $\left(E \otimes F, h_{E} h_{\varepsilon}\right)$ in the proof of Theorem [5.1, where $\left\{h_{\varepsilon}\right\}_{1 \gg \varepsilon>0}$ is a family of singular Hermitian metrics on $F$ (constructed in Step 11) and $h_{E}$ is a smooth Hermitian metric on $E$ such that $\sqrt{-1} \Theta_{h_{E}}(E)$ is Nakano semipositive. Then it is easy to see that essentially the same proof as in Theorem 5.1 works for Theorem 6.6 thanks to the assumption on the curvature of $E$. For the reader's convenience, we give several remarks on the differences with the proof of Theorem 5.1.

There is no problem when we construct $h_{\varepsilon}$ and $\omega_{\varepsilon, \delta}$. In Step 4 in the proof of Theorem [5.1, we used the de Rham-Weil isomorphism (see (5.7) and [MaS4, Proposition 5.5]), which was constructed by using Lemma 5.12, Since [D1, 4.1 Théorème] (which yields Lemma 5.12) is formulated for holomorphic vector bundles, Lemma 5.12 can be generalized to $\left(E \otimes F, h_{E} h_{\varepsilon}\right)$. From this generalization, we can construct the de Rham-Weil isomorphism for $E \otimes F$

$\overline{f_{\varepsilon, \delta}}: \frac{\operatorname{Ker} \bar{\partial}}{\operatorname{Im} \bar{\partial}}$ of $L_{(2)}^{n, q}(E \otimes F)_{h_{E} h_{\varepsilon}, \omega_{\varepsilon, \delta}} \cong \frac{\operatorname{Ker} \mu}{\operatorname{Im} \mu}$ of $C^{q}\left(\mathcal{U}, K_{X} \otimes E \otimes F \otimes \mathcal{J}\left(h_{\varepsilon}\right)\right)$.

In Step 1, we used the orthogonal decomposition of $L_{(2)}^{n, q}(F)_{\varepsilon, \delta}$, which was obtained from the fact that $\operatorname{Im} \bar{\partial} \subset L_{(2)}^{n, q}(F)_{\varepsilon, \delta}$ is closed. To obtain the same conclusion for $L_{(2)}^{n, q}(E \otimes F)_{h_{E} h_{\varepsilon}, \omega_{\varepsilon, \delta}}$, it is sufficient to show that $C^{q}\left(\mathcal{U}, K_{X} \otimes E \otimes F \otimes \mathcal{J}\left(h_{\varepsilon}\right)\right)$ is a Fréchet space (see [MaS4, Proposition 5.8]). We can easily check it by using the same argument as in MaS4, Theorem 5.3] for $\mathbb{C}^{\mathrm{rank} E}$-valued holomorphic functions.

The argument of Step 2 works even if we consider $\left(E \otimes F, h_{E} h_{\varepsilon}\right)$. In Step 3, we need to prove (5.6), but it is easy to see

$$
\begin{aligned}
-\varepsilon q\left|u_{\varepsilon, \delta}\right|_{h_{E} h_{\varepsilon}, \omega_{\varepsilon, \delta}}^{2} & \leq\left\langle\sqrt{-1} \Theta_{h_{\varepsilon}}(F) \Lambda_{\omega_{\varepsilon, \delta}} u_{\varepsilon, \delta}, u_{\varepsilon, \delta}\right\rangle_{h_{E} h_{\varepsilon}, \omega_{\varepsilon, \delta}} \\
& \leq\left\langle\sqrt{-1} \Theta_{h_{E} h_{\varepsilon}}(E \otimes F) \Lambda_{\omega_{\varepsilon, \delta}} u_{\varepsilon, \delta}, u_{\varepsilon, \delta}\right\rangle_{h_{E} h_{\varepsilon}, \omega_{\varepsilon, \delta}}
\end{aligned}
$$

since $\sqrt{-1} \Theta_{h_{E}}(E)$ is Nakano semipositive.

When $E$ is Nakano semipositive and is not flat, there seems to be no Hodge theoretic approach to Theorem 6.6 even if $h$ is smooth.

\section{ACKNOWLEDGMENTS}

The authors would like to thank Professor Toshiyuki Sugawa for giving them the reference on Example 3.10 and Professor Taro Fujisawa for his warm encouragement. Furthermore, they are deeply grateful to Professor Sébastien Boucksom for kindly suggesting Problem 1.11. The first author thanks Takahiro Shibata for discussion.

\section{REFERENCES}

[AK] Allen Altman and Steven Kleiman, Introduction to Grothendieck duality theory, Lecture Notes in Mathematics, Vol. 146, Springer-Verlag, Berlin-New York, 1970. MR.0274461

[A1] F. Ambro, Quasi-log varieties, Tr. Mat. Inst. Steklova 240 (2003), no. Biratsion. Geom. Lineı̆n. Sist. Konechno Porozhdennye Algebry, 220-239; English transl., Proc. Steklov Inst. Math. 1(240) (2003), 214-233. MR.1993751 
[A2] Florin Ambro, An injectivity theorem, Compos. Math. 150 (2014), no. 6, 999-1023, DOI 10.1112/S0010437X13007768. MR3223880

[Ar] Donu Arapura, A note on Kollár's theorem, Duke Math. J. 53 (1986), no. 4, 1125-1130, DOI 10.1215/S0012-7094-86-05355-X. MR874684

[Be] Bo Berndtsson, Prekopa's theorem and Kiselman's minimum principle for plurisubharmonic functions, Math. Ann. 312 (1998), no. 4, 785-792, DOI 10.1007/s002080050246. MR.1660227

[CDM] JunYan Cao, Jean-Pierre Demailly, and Shin-ichi Matsumura, A general extension theorem for cohomology classes on non reduced analytic subspaces, Sci. China Math. 60 (2017), no. 6, 949-962, DOI 10.1007/s11425-017-9066-0. MR3647124

[D1] Jean-Pierre Demailly, Estimations $L^{2}$ pour l'opérateur $\bar{\partial}$ d'un fibré vectoriel holomorphe semi-positif au-dessus d'une variété kählérienne complète (French), Ann. Sci. École Norm. Sup. (4) 15 (1982), no. 3, 457-511. MR690650

[D2] Jean-Pierre Demailly, A numerical criterion for very ample line bundles, J. Differential Geom. 37 (1993), no. 2, 323-374. MR1205448

[D3] Jean-Pierre Demailly, Analytic methods in algebraic geometry, Surveys of Modern Mathematics, vol. 1, International Press, Somerville, MA; Higher Education Press, Beijing, 2012. MR2978333

[D4] J.-P. Demailly, Complex analytic and differential geometry, available at the web page of the author. https://www-fourier.ujf-grenoble.fr/ demailly/manuscripts/agbook. pdf

[DHP] Jean-Pierre Demailly, Christopher D. Hacon, and Mihai Păun, Extension theorems, nonvanishing and the existence of good minimal models, Acta Math. 210 (2013), no. 2, 203259, DOI 10.1007/s11511-013-0094-x. MR3070567

[DPS] Jean-Pierre Demailly, Thomas Peternell, and Michael Schneider, Pseudo-effective line bundles on compact Kähler manifolds, Internat. J. Math. 12 (2001), no. 6, 689-741, DOI 10.1142/S0129167X01000861. MR1875649

[En] Ichiro Enoki, Kawamata-Viehweg vanishing theorem for compact Kähler manifolds, Einstein metrics and Yang-Mills connections (Sanda, 1990), Lecture Notes in Pure and Appl. Math., vol. 145, Dekker, New York, 1993, pp. 59-68. MR.1215279

[EV] Hélène Esnault and Eckart Viehweg, Lectures on vanishing theorems, DMV Seminar, vol. 20, Birkhäuser Verlag, Basel, 1992, DOI 10.1007/978-3-0348-8600-0. MR1193913

[F1] Osamu Fujino, On injectivity, vanishing and torsion-free theorems for algebraic varieties, Proc. Japan Acad. Ser. A Math. Sci. 85 (2009), no. 8, 95-100, DOI 10.3792/pjaa.85.95. MR2561896

[F2] Osamu Fujino, Introduction to the theory of quasi-log varieties, Classification of algebraic varieties, EMS Ser. Congr. Rep., Eur. Math. Soc., Zürich, 2011, pp. 289-303, DOI 10.4171/007-1/13. MR2779477

[F3] Osamu Fujino, Fundamental theorems for the log minimal model program, Publ. Res. Inst. Math. Sci. 47 (2011), no. 3, 727-789, DOI 10.2977/PRIMS/50. MR2832805

[F4] Osamu Fujino, A transcendental approach to Kollár's injectivity theorem, Osaka J. Math. 49 (2012), no. 3, 833-852. MR 2993068

[F5] Osamu Fujino, A transcendental approach to Kollár's injectivity theorem II, J. Reine Angew. Math. 681 (2013), 149-174, DOI 10.1515/crelle-2012-0036. MR3181493

[F6] Osamu Fujino, Vanishing theorems, Minimal models and extremal rays (Kyoto, 2011), Adv. Stud. Pure Math., vol. 70, Math. Soc. Japan, [Tokyo], 2016, pp. 299-321, DOI 10.2969/aspm/07010299. MR3618264

[F7] Osamu Fujino, Injectivity theorems, Higher dimensional algebraic geometry - in honour of Professor Yujiro Kawamata's sixtieth birthday, Adv. Stud. Pure Math., vol. 74, Math. Soc. Japan, Tokyo, 2017, pp. 131-157, DOI 10.2969/aspm/07410131. MR3791211

[F8] Osamu Fujino, Fundamental theorems for semi log canonical pairs, Algebr. Geom. 1 (2014), no. 2, 194-228, DOI 10.14231/AG-2014-011. MR3238112

[F9] Osamu Fujino, Foundations of the minimal model program, MSJ Memoirs, vol. 35, Mathematical Society of Japan, Tokyo, 2017. MR3643725

[F10] Osamu Fujino, Kodaira vanishing theorem for log-canonical and semi-log-canonical pairs, Proc. Japan Acad. Ser. A Math. Sci. 91 (2015), no. 8, 112-117, DOI 10.3792/pjaa.91.112. MR. 3403942 
[F11] Osamu Fujino, On semipositivity, injectivity and vanishing theorems, Hodge theory and $L^{2}$-analysis, Adv. Lect. Math. (ALM), vol. 39, Int. Press, Somerville, MA, 2017, pp. 245282. MR 3751293

[F12] Osamu Fujino, Vanishing and semipositivity theorems for semi-log canonical pairs, Publ. Res. Inst. Math. Sci. 56 (2020), no. 1, 15-32, DOI 10.4171/prims/56-1-2. MR4055972

[F13] Osamu Fujino, Effective basepoint-free theorem for semi-log canonical surfaces, Publ. Res. Inst. Math. Sci. 53 (2017), no. 3, 349-370, DOI 10.4171/PRIMS/53-3-1. MR3669739

[F14] Osamu Fujino, Kollár-type effective freeness for quasi-log canonical pairs, Internat. J. Math. 27 (2016), no. 14, 1650114, 15, DOI 10.1142/S0129167X16501147. MR3593676

[F15] Osamu Fujino, Kollár-Nadel type vanishing theorem, Southeast Asian Bull. Math. 42 (2018), no. 5, 643-646. MR 3888433

[F16] O. Fujino, Relative Bertini type theorem for multiplier ideal sheaves, preprint, arXiv:1709.01406 [math.AG], 2017.

[Fs] Taro Fujisawa, A remark on the decomposition theorem for direct images of canonical sheaves tensorized with semipositive vector bundles, Proc. Japan Acad. Ser. A Math. Sci. 92 (2016), no. 7, 84-85, DOI 10.3792/pjaa.92.84. MR 3529090

[GM] Yoshinori Gongyo and Shin-ichi Matsumura, Versions of injectivity and extension theorems (English, with English and French summaries), Ann. Sci. Éc. Norm. Supér. (4) 50 (2017), no. 2, 479-502, DOI 10.24033/asens.2325. MR3621435

[GL] Mark Green and Robert Lazarsfeld, Deformation theory, generic vanishing theorems, and some conjectures of Enriques, Catanese and Beauville, Invent. Math. 90 (1987), no. 2, 389-407, DOI 10.1007/BF01388711. MR910207

[Ha] Christopher D. Hacon, A derived category approach to generic vanishing, J. Reine Angew. Math. 575 (2004), 173-187, DOI 10.1515/crll.2004.078. MR2097552

[Hö] Andreas Höring, Positivity of direct image sheaves - a geometric point of view, Enseign. Math. (2) 56 (2010), no. 1-2, 87-142, DOI 10.4171/LEM/56-1-4. MR2674856

[Kod] K. Kodaira, On a differential-geometric method in the theory of analytic stacks, Proc. Nat. Acad. Sci. U.S.A. 39 (1953), 1268-1273, DOI 10.1073/pnas.39.12.1268. MR66693

[Kol1] János Kollár, Higher direct images of dualizing sheaves. I, Ann. of Math. (2) 123 (1986), no. 1, 11-42, DOI 10.2307/1971351. MR825838

[Kol2] János Kollár, Shafarevich maps and automorphic forms, M. B. Porter Lectures, Princeton University Press, Princeton, NJ, 1995, DOI 10.1515/9781400864195. MR1341589

[L1] Robert Lazarsfeld, Positivity in algebraic geometry. I, Ergebnisse der Mathematik und ihrer Grenzgebiete. 3. Folge. A Series of Modern Surveys in Mathematics [Results in Mathematics and Related Areas. 3rd Series. A Series of Modern Surveys in Mathematics], vol. 48, Springer-Verlag, Berlin, 2004. Classical setting: line bundles and linear series, DOI 10.1007/978-3-642-18808-4. MR2095471

[L2] Robert Lazarsfeld, Positivity in algebraic geometry. II, Ergebnisse der Mathematik und ihrer Grenzgebiete. 3. Folge. A Series of Modern Surveys in Mathematics [Results in Mathematics and Related Areas. 3rd Series. A Series of Modern Surveys in Mathematics], vol. 49, Springer-Verlag, Berlin, 2004. Positivity for vector bundles, and multiplier ideals, DOI 10.1007/978-3-642-18808-4. MR2095472

[L3] Robert Lazarsfeld, A short course on multiplier ideals, Analytic and algebraic geometry, IAS/Park City Math. Ser., vol. 17, Amer. Math. Soc., Providence, RI, 2010, pp. 451-494. MR2743821

[LP] Vladimir Lazić and Thomas Peternell, Abundance for varieties with many differential forms, Épijournal Géom. Algébrique 2 (2018), Art. 1, 35, DOI 10.46298/epiga.2018.volume2.3867. MR3770541

[LRW] Kefeng Liu, Sheng Rao, and Xueyuan Wan, Geometry of logarithmic forms and deformations of complex structures, J. Algebraic Geom. 28 (2019), no. 4, 773-815, DOI 10.1090/jag/723. MR3994313

[Man] Mirella Manaresi, Sard and Bertini type theorems for complex spaces, Ann. Mat. Pura Appl. (4) 131 (1982), 265-279, DOI 10.1007/BF01765156. MR681567

$[\mathrm{MaH}]$ Hideyuki Matsumura, Commutative ring theory, 2nd ed., Cambridge Studies in Advanced Mathematics, vol. 8, Cambridge University Press, Cambridge, 1989. Translated from the Japanese by M. Reid. MR 1011461

[MaS1] Shin-ichi Matsumura, A Nadel vanishing theorem via injectivity theorems, Math. Ann. 359 (2014), no. 3-4, 785-802, DOI 10.1007/s00208-014-1018-6. MR3231016 
[MaS2] Shin-ichi Matsumura, A Nadel vanishing theorem for metrics with minimal singularities on big line bundles, Adv. Math. 280 (2015), 188-207, DOI 10.1016/j.aim.2015.03.019. MR.3350216

[MaS3] Shin-ichi Matsumura, Some applications of the theory of harmonic integrals, Complex Manifolds 2 (2015), no. 1, 16-25, DOI 10.1515/coma-2015-0003. MR3370350

[MaS4] Shin-ichi Matsumura, An injectivity theorem with multiplier ideal sheaves of singular metrics with transcendental singularities, J. Algebraic Geom. 27 (2018), no. 2, 305-337, DOI $10.1090 / \mathrm{jag} / 687$. MR.3764278

[MaS5] S. Matsumura, An injectivity theorem with multiplier ideal sheaves for higher direct images under Kähler morphisms, to appear in Algebr. Geom. arXiv:1607.05554v2, 2015.

[MaS6] Shin-ichi Matsumura, A vanishing theorem of Kollár-Ohsawa type, Math. Ann. 366 (2016), no. 3-4, 1451-1465, DOI 10.1007/s00208-016-1371-8. MR3563242

[MaS7] Shin-ichi Matsumura, Injectivity theorems with multiplier ideal sheaves and their applications, Complex analysis and geometry, Springer Proc. Math. Stat., vol. 144, Springer, Tokyo, 2015, pp. 241-255, DOI 10.1007/978-4-431-55744-9_18. MR3446761

[MaS8] Shin-ichi Matsumura, A transcendental approach to injectivity theorem for log canonical pairs, Ann. Sc. Norm. Super. Pisa Cl. Sci. (5) 19 (2019), no. 1, 311-334. MR3923849

[Mo] Christophe Mourougane, Théorèmes d'annulation générique pour les fibrés vectoriels semi-négatifs (French, with English and French summaries), Bull. Soc. Math. France 127 (1999), no. 1, 115-133. MR.1700471

[No] Junjiro Noguchi, A short analytic proof of closedness of logarithmic forms, Kodai Math. J. 18 (1995), no. 2, 295-299, DOI 10.2996/kmj/1138043426. MR1346909

[O1] Takeo Ohsawa, On complete Kähler domains with $C^{1}$-boundary, Publ. Res. Inst. Math. Sci. 16 (1980), no. 3, 929-940, DOI 10.2977/prims/1195186937. MR602476

[O2] Takeo Ohsawa, Vanishing theorems on complete Kähler manifolds, Publ. Res. Inst. Math. Sci. 20 (1984), no. 1, 21-38, DOI 10.2977/prims/1195181825. MR736089

[O3] Takeo Ohsawa, On a curvature condition that implies a cohomology injectivity theorem of Kollár-Skoda type, Publ. Res. Inst. Math. Sci. 41 (2005), no. 3, 565-577. MR2153535

[OT] Takeo Ohsawa and Kenshō Takegoshi, On the extension of $L^{2}$ holomorphic functions, Math. Z. 195 (1987), no. 2, 197-204, DOI 10.1007/BF01166457. MR892051

[PP] Giuseppe Pareschi and Mihnea Popa, GV-sheaves, Fourier-Mukai transform, and generic vanishing, Amer. J. Math. 133 (2011), no. 1, 235-271, DOI 10.1353/ajm.2011.0000. MR.2752940

[Pa] Mihai Păun, Siu's invariance of plurigenera: a one-tower proof, J. Differential Geom. 76 (2007), no. 3, 485-493. MR2331528

[Ra] Thomas Ransford, Potential theory in the complex plane, London Mathematical Society Student Texts, vol. 28, Cambridge University Press, Cambridge, 1995, DOI 10.1017/CBO9780511623776. MR1334766

[Sc] C. Schnell, A graduate course on the generic vanishing theorem (Stony Brook and Bonn, 2013), available at the web page of the author. http://www.math.stonybrook. edu/ cschnell

[Si] Yum-Tong Siu, Extension of twisted pluricanonical sections with plurisubharmonic weight and invariance of semipositively twisted plurigenera for manifolds not necessarily of general type, Complex geometry (Göttingen, 2000), Springer, Berlin, 2002, pp. 223-277. MR.1922108

[Ta] Kenshō Takegoshi, Higher direct images of canonical sheaves tensorized with semi-positive vector bundles by proper Kähler morphisms, Math. Ann. 303 (1995), no. 3, 389-416, DOI 10.1007/BF01460997. MR1354997

[Wu Lei Wu, Vanishing and injectivity theorems for Hodge modules, Trans. Amer. Math. Soc. 369 (2017), no. 11, 7719-7736, DOI 10.1090/tran/6869. MR3695842

[ZZ] Xiangyu Zhou and Langfeng Zhu, Regularization of quasi-plurisubharmonic functions on complex manifolds, Sci. China Math. 61 (2018), no. 7, 1163-1174, DOI 10.1007/s11425018-9289-4. MR3817169 
Department of Mathematics, Graduate School of Science, Kyoto University, Kyoto 606-8502, JAPAN

Email address: fujino@math.kyoto-u.ac.jp

Mathematical Institute, Tohoku University, 6-3, Aramaki Aza-Aoba, Aoba-ku, Sendai 980-8578, JAPAN.

Email address: mshinichi-math@tohoku.ac.jp, mshinichio@gmail.com 